\title{
Integral formulations of the geometric eikonal equation
}

\author{
AuréLIEN MonteILleT ${ }^{\dagger}$ \\ UFR Sciences et Techniques, Université de Bretagne Occidentale, \\ 6 av. Le Gorgeu, BP 809, 29285 Brest, France
}

[Received 23 February 2006 and in revised form 14 November 2006]

\begin{abstract}
We prove integral formulations of the eikonal equation $u_{t}=c(x, t)|D u|$, equivalent to the notion of viscosity solution in the framework of the set-theoretic approach to front propagation problems. We apply these integral formulations to investigate the regularity of the front: we prove that under regularity assumptions on the velocity $c$, the front has locally finite perimeter in $\{c \neq 0\}$, and we give a time-integral estimate of its perimeter.
\end{abstract}

Keywords: Eikonal equation; viscosity solutions; set-theoretic approach; functions of bounded variation; sets of finite perimeter.

\section{Introduction}

We are interested in generalized time evolutions of subsets $K(t)$ of $\mathbb{R}^{N}, N \geqslant 1$, governed by the following geometric law:

$$
V_{x, t}=c(x, t),
$$

where $V_{x, t}$ denotes the normal velocity of a point $x$ of $\partial K(t)$ at time $t$. If for example $K(t)$ can be represented by

$$
K(t)=\left\{x \in \mathbb{R}^{N} ; u(x, t) \geqslant 0\right\}, \quad \partial K(t)=\left\{x \in \mathbb{R}^{N} ; u(x, t)=0\right\}
$$

for some $C^{1}$ function $u: \mathbb{R}^{N} \times[0, T] \rightarrow \mathbb{R}$ such that $u(x, t)=0$ implies that the space gradient $D u(x, t) \neq 0$, then a classical calculation yields

$$
V_{x, t}=\frac{u_{t}(x, t)}{|D u(x, t)|},
$$

where $u_{t}$ denotes the time derivative of $u$, and $|\cdot|$ the usual euclidian norm on $\mathbb{R}^{N}\left(|x|=\langle x, x\rangle^{1 / 2}\right)$, so that $u$ satisfies the so-called eikonal equation

$$
u_{t}=c(x, t)|D u|
$$

for all $(x, t) \in \mathbb{R}^{N} \times(0, T)$ such that $u(x, t)=0$. If the front $\partial K(t)$ is not assumed to be regular, equation (1.2) gives a generalized way of studying the evolution, and the notion of viscosity solution of this equation provides a satisfactory framework to do so. More precisely, one possible generalized solution of the geometric evolution (1.1), called a set-theoretic solution, is a family $(K(t))$ of subsets of $\mathbb{R}^{N}$ such that $(x, t) \mapsto \mathbf{1}_{K(t)}(x)$ is a discontinuous viscosity solution of (1.2), where $\mathbf{1}_{E}$ is the indicator function of a set $E$. We refer to [13] for a complete overview of viscosity solutions, and to

\footnotetext{
${ }^{\dagger}$ E-mail: aurelien.monteillet@univ-brest.fr
} 
[17] for details about set-theoretic solutions. Various applications of the notion of viscosity solutions can also be found in [5, 6, 7, 14, 19], to mention but a few. Other approaches to the problems of propagating fronts are presented in [2, 10, 21].

Our work is motivated by a model for the dynamics of dislocation lines in a crystal, which gives rise to a Hamilton-Jacobi equation with a non-local term. In questions related to existence for this non-local equation, it is interesting to understand the behavior of the solutions of the eikonal equation, and especially the regularity properties of the front $\partial K(t)$. This has been done for example by Evans and Spruck [16] for the motion by mean curvature. In the case of the geometric eikonal equation, the regularity of the front has recently been investigated for positive velocities by Alvarez, Cardaliaguet and Monneau [1], and using another method by Barles and Ley [8], and their works show that a good regularity property to investigate is that of finite perimeter. More precisely, it is proved in [1] that the $K(t)$ 's have finite perimeter if $c>0$ and $K(0)$ has the interior ball property, i.e. is the union of closed balls of some fixed radius. Cardaliaguet and Cannarsa [11] then improved and generalized the estimates of [1]. However, results in this spirit lack for velocities with no sign.

To achieve such results, we search for analytic ways of studying the geometric equation (1.1, and we first focus on the case of set-theoretic subsolutions. One clue in this direction is Hadamard's formula in the case where $K(t)$ is open, bounded, and $(K(t))$ evolves smoothly in time: for all $\phi \in C^{1}\left(\mathbb{R}^{N} \times[0, T], \mathbb{R}\right)$,

$$
\frac{\mathrm{d}}{\mathrm{d} t} \int_{K(t)} \phi(x, t) \mathrm{d} x=\int_{K(t)} \phi_{t}(x, t) \mathrm{d} x+\int_{\partial K(t)} V_{x, t} \phi(x, t) \mathrm{d} \mathcal{H}^{N-1}(x),
$$

where $\mathcal{H}^{N-1}$ denotes the $(N-1)$-dimensional Hausdorff measure. In particular, if $V_{x, t} \leqslant c(x, t)$ in the classical sense, then for all $\phi \in C^{1}\left(\mathbb{R}^{N} \times[0, T], \mathbb{R}_{+}\right)$, we have

$$
\frac{\mathrm{d}}{\mathrm{d} t} \int_{K(t)} \phi(x, t) \mathrm{d} x \leqslant \int_{K(t)} \phi_{t}(x, t) \mathrm{d} x+\int_{\partial K(t)} c(x, t) \phi(x, t) \mathrm{d} \mathcal{H}^{N-1}(x) .
$$

The problem is that the term $\int_{\partial K(t)} c(x, t) \phi(x, t) \mathrm{d} \mathcal{H}^{N-1}(x)$ does not make sense in the viscosity solution framework: the regularity of $K(t)$ in terms of perimeter is unknown (actually, one of the reasons to consider this formula is to give a meaning to this term). However, if we set

$$
K^{\varepsilon}(t)=\left\{x \in \mathbb{R}^{N} ; \mathrm{d}_{K(t)}(x)<\varepsilon\right\},
$$

where $d_{F}$ denotes the distance function to some closed non-empty set $F \subset \mathbb{R}^{N}$, then $K^{\varepsilon}(t)$ has finite perimeter and one of our main results is that $(1.3)$ admits the following integral generalization: under standard regularity assumptions on the velocity $c: \mathbb{R}^{N} \times[0, T] \rightarrow \mathbb{R}$, we have for all $t_{1}$ and $t_{2}$ satisfying $0 \leqslant t_{1} \leqslant t_{2} \leqslant T$, for almost all $\varepsilon>0$, and for all $\phi \in C^{1}\left(\mathbb{R}^{N} \times[0, T], \mathbb{R}_{+}\right)$,

$$
\left[\int_{K^{\varepsilon}(t)} \phi(x, t) \mathrm{d} x\right]_{t_{1}}^{t_{2}} \leqslant \int_{t_{1}}^{t_{2}} \int_{K^{\varepsilon}(t)} \phi_{t}(x, t) \mathrm{d} x \mathrm{~d} t+\int_{t_{1}}^{t_{2}} \int_{\left\{d_{K(t)}=\varepsilon\right\}} c^{\varepsilon}(x, t) \phi(x, t) \mathrm{d} \mathcal{H}^{N-1}(x) \mathrm{d} t,
$$

where we have introduced a perturbation of the velocity by

$$
c^{\varepsilon}(x, t)=\max _{|y-x| \leqslant \varepsilon} c(y, t) .
$$

We find in [1], in the case where $c>0$, an integral formulation valid for any family $(K(t))$ such that $\mathbf{1}_{K(t)}$ satisfies the eikonal equation almost everywhere. In our case, although the perturbation 
by $\varepsilon$ seems restrictive at first glance, we have in compensation considerable freedom in choosing $\varepsilon$. As a consequence, we will prove that our integral formulation is actually equivalent to the notion of set-theoretic subsolution of the eikonal equation.

After setting the notations in Section 2, we prove the integral formulation of the eikonal equation for subsolutions (Theorem 3.1) in Section 3. Conversely, we prove in Section 4 that this integral formulation characterizes the notion of set-theoretic subsolution of (1.1): this is Theorem 4.1 Then in Section 5 we use the integral formulation for subsolutions to investigate regularity properties of the $K(t)$ 's and give a time-integral estimate of their perimeter. These results are contained in Theorem 5.1. All the results can be adapted to the notion of set-theoretic supersolutions, as expected. We collect the corresponding results in Section 6. Finally, in Section 7, under a regularity assumption on the evolution related to the non-empty interior difficulty arising in the level-set method, we combine these results for sub- and supersolutions to prove in Theorem 7.1 that for almost all $t \in[0, T], K(t)$ has locally finite perimeter in $\{c(\cdot, t) \neq 0\}$, and that we have the following estimate:

$$
\begin{aligned}
& \int_{0}^{T} \int_{\partial^{*} K(t)}|c(x, t)| \mathrm{d} \mathcal{H}^{N-1}(x) \mathrm{d} t \\
& \quad \leqslant\left[\int_{K(t)} \operatorname{sgn}(c(x, t)) \mathrm{d} x\right]_{0}^{T}+2 \int_{0}^{T} \int_{K(t) \cap\{c(, t)=0\}} \frac{\left|c_{t}(x, t)\right|}{|D c(x, t)|} \mathbf{1}_{\left\{c_{t}<0\right\}} \mathrm{d} \mathcal{H}^{N-1}(x) \mathrm{d} t,
\end{aligned}
$$

where $\partial^{*} K(t)$ is the reduced boundary of $K(t)$, and $\operatorname{sgn}(c)$ the sign of $c$.

\section{Notations and tools}

Let us start with standard notations. $B(x, r)$ (resp. $\bar{B}(x, r))$ denotes the open (resp. closed) ball of radius $r>0$ centered at $x \in \mathbb{R}^{N}$. The notation $E^{c}$ stands for the complement of a set $E$, and $\mathcal{L}^{p}$ denotes the Lebesgue measure on $\mathbb{R}^{p}$. Finally, $C^{0}(U, V)$ (resp. $C^{1}(U, V)$ ) is the set of continuous (resp. continuously differentiable) functions from $U \subset \mathbb{R}^{p}$ to $V \subset \mathbb{R}^{q}$. We add a subscript $c$ to indicate that in addition these functions have compact support in $U: C_{c}^{0}(U, V), C_{c}^{1}(U, V)$. Finally, we abbreviate "upper semicontinuous" (resp. "lower semicontinuous") to usc (resp. lsc).

\subsection{Viscosity solutions and set-theoretic approach}

We first give the results that ensure the existence of set-theoretic sub- and supersolutions of the eikonal equation with initial value:

$$
\begin{cases}u_{t}=c(x, t)|D u| & \text { in } \mathbb{R}^{N} \times(0, T), \\ u(x, 0)=u_{0}(x) & \text { in } \mathbb{R}^{N} .\end{cases}
$$

For the convenience of the reader, we recall the definitions of a viscosity sub- and supersolution of the eikonal equation and of a solution of problem 2.1]:

Definition 2.1 (Crandall and Lions [14]) 1 . We say that $u: \mathbb{R}^{N} \times[0, T] \rightarrow \mathbb{R}$ is a viscosity subsolution (resp. supersolution) of $u_{t}=c(x, t)|D u|$ in $\mathbb{R}^{N} \times(0, T)$ if $u$ is usc (resp. lsc) and if for all test functions $\phi$ of class $C^{1}$ such that $u-\phi$ has a local maximum (resp. minimum) at $(x, t) \in \mathbb{R}^{N} \times(0, T)$,

$$
\phi_{t}(x, t) \leqslant c(x, t)|D \phi(x, t)| \quad(\text { resp. } \geqslant) .
$$


2. We say that $u: \mathbb{R}^{N} \times[0, T] \rightarrow \mathbb{R}$ is a viscosity solution of (2.1) if $u$ is both a viscosity sub- and supersolution of $u_{t}=c(x, t)|D u|$ in $\mathbb{R}^{N} \times(0, T)$ and if $u(\cdot, 0)=u_{0}$.

THEOREM 2.2 (Crandall and Lions [14]) Suppose that $c: \mathbb{R}^{N} \times[0, T] \rightarrow \mathbb{R}$ is continuous, bounded, and Lipschitz continuous with respect to the space variable. Then the problem 2.1 has a unique uniformly continuous viscosity solution $u: \mathbb{R}^{N} \times[0, T] \rightarrow \mathbb{R}$ for every uniformly continuous initial value $u_{0}$.

Let $K(0)=\overline{\Omega(0)}$ be fixed with $\Omega(0)$ open, $K(0) \neq \emptyset, \mathbb{R}^{N}$, and let $\bar{d}_{K(0)}$ denote the signed distance function to $K(0)$,

$$
\bar{d}_{K(0)}(x)= \begin{cases}d_{\partial K(0)}(x) & \text { if } x \in K(0) \\ -d_{K(0)}(x) & \text { if } x \notin K(0) .\end{cases}
$$

Let $u$ be the solution of 2.1 with initial value $u_{0}=\bar{d}_{K(0)}$. A technique adapted from Barles, Soner and Souganidis ([9, Theorem 2.1]), which relies on the stability theorem for viscosity solutions, shows that $\mathbf{1}_{\{u \geqslant 0\}}$ is also a subsolution of $[1.2\}$, while $\mathbf{1}_{\{u>0\}}$ is a supersolution. Thus if we set $K(t)=\left\{x \in \mathbb{R}^{N} ; u(x, t) \geqslant 0\right\}$, and $\Omega(t)=\left\{x \in \mathbb{R}^{N} ; u(x, t)>0\right\}$, we obtain a new subsolution $\underline{u}:(x, t) \mapsto \mathbf{1}_{K(t)}(x)$ and a new supersolution $\bar{u}:(x, t) \mapsto \mathbf{1}_{\Omega(t)}(x)$ of $[1.2)$ in $\mathbb{R}^{N} \times(0, T)$, with respective initial values $\mathbf{1}_{K(0)}$ and $\mathbf{1}_{\Omega(0)}$.

We notice that the upper semicontinuity of $\underline{u}$ on $\mathbb{R}^{N} \times[0, T]$ required in the definition of subsolution is equivalent to the fact that the graph of $K: t \mapsto K(t)$, i.e. $\operatorname{Graph}(K)=$ $\bigcup_{t \in[0, T]}\{t\} \times K(t)$, is closed in $[0, T] \times \mathbb{R}^{N}$. Likewise, the lower semicontinuity of $\bar{u}$ on $\mathbb{R}^{N} \times[0, T]$ is equivalent to the graph of $\Omega: t \mapsto \Omega(t)$ being open in $[0, T] \times \mathbb{R}^{N}$.

From now on we are going to focus on these so-called set-theoretic sub- and supersolutions of the form $\underline{u}:(x, t) \mapsto \mathbf{1}_{K(t)}(x)$ and $\bar{u}:(x, t) \mapsto \mathbf{1}_{\Omega(t)}(x)$ respectively. We first gather some properties of the geometric evolutions $t \mapsto K(t)$ and $t \mapsto \Omega(t)$. We will write $\Omega^{c}(t)$ for $(\Omega(t))^{c}$.

PROPOSITION 2.3 Let $c$ satisfy the assumptions of Theorem 2.2. and let $L=\|c\|_{\infty}$.

(i) Let $K:[0, T] \rightarrow \mathcal{P}\left(\mathbb{R}^{N}\right) \backslash\{\emptyset\}$ be such that $\underline{u}:(x, t) \mapsto \mathbf{1}_{K(t)}(x)$ is a subsolution of (1.2) in $\mathbb{R}^{N} \times(0, T)$. Then for all $t \in[0, T]$ and for all $s \in[0, T-t)$, we have $K(t+s) \subset$ $K(t)+\bar{B}(0, L s)$. In particular, the evolution is bounded on $[0, T)$ if $K(0)$ is compact.

(ii) Let $\Omega:[0, T] \rightarrow \mathcal{P}\left(\mathbb{R}^{N}\right) \backslash\left\{\mathbb{R}^{N}\right\}$ be such that $\bar{u}:(x, t) \mapsto \mathbf{1}_{\Omega(t)}(x)$ is a supersolution of 1.2) in $\mathbb{R}^{N} \times(0, T)$. Then for all $t \in[0, T]$ and for all $s \in[0, T-t)$, we have $\Omega^{c}(t+s) \subset$ $\Omega^{c}(t)+\bar{B}(0, L s)$.

Proof. Let us prove (i). Since $\underline{u}:(x, t) \mapsto \mathbf{1}_{K(t)}(x)$ is a viscosity subsolution of $u_{t}=c(x, t)|D u|$, we deduce from the inequality $|c| \leqslant L$ that for any fixed $t \in[0, T), u_{1}:(x, s) \mapsto \underline{u}(x, t+s)$ is a subsolution of $u_{t}=L|D u|$ on $\mathbb{R}^{N} \times(0, T-t)$ with $u_{1}(\cdot, 0)=\mathbf{1}_{K(t)}$. The solution of this last equation with initial value $u_{2}(\cdot, 0): x \mapsto \max \left(1-d_{K(t)}(x), 0\right)$, which is uniformly continuous, is known and given by $u_{2}:(x, s) \mapsto \max _{|y-x| \leqslant L s} u_{2}(y, 0)$. Since $u_{1}$ and $u_{2}$ satisfy $u_{1}(\cdot, 0) \leqslant$ $u_{2}(\cdot, 0)$, we deduce from the comparison principle (see [9. Theorem 1.3]) that for all $(x, s) \in \mathbb{R}^{N} \times$ $[0, T-t)$,

$$
u_{1}(x, s) \leqslant u_{2}(x, s), \quad \text { i.e. } \quad \underline{u}(x, t+s) \leqslant \max _{|y-x| \leqslant L s} u_{2}(y, 0) .
$$

Therefore if $x \notin K(t)+\bar{B}(0, L s)$, then for all $y$ with $|y-x| \leqslant L s, y \notin K(t)$, i.e. $u_{2}(y, 0)<1$. As a result $\underline{u}(x, t+s)<1$, that is, $x \notin K(t+s)$. 
(ii) follows from (i) since it is straightforward to check that $(x, t) \mapsto \mathbf{1}_{\Omega^{c}(t)}(x)=1-\bar{u}(x, t)$ is a subsolution of $u_{t}=-c(x, t)|D u|$ in $\mathbb{R}^{N} \times(0, T)$. However, we point out that we cannot deduce from this property on $\Omega^{c}(t)$ that the evolution $t \mapsto \Omega(t)$ is bounded on $[0, T)$ if $\Omega(0)$ is bounded.

We now state properties of regularity in time of the distance function to the fronts:

Proposition 2.4 Under the assumptions of Proposition 2.3. $(x, t) \mapsto d_{K(t)}(x)$ is lsc on $\mathbb{R}^{N} \times$ $[0, T]$, and for all $x \in \mathbb{R}^{N}, t \mapsto d_{K(t)}(x)$ is left continuous on $(0, T)$. The same conclusions hold for $(x, t) \mapsto d_{\Omega^{c}(t)}(x)$.

Proof. We only treat the case of $K$ since the proof for $\Omega$ is the same.

1. Let us show that $(x, t) \mapsto d_{K(t)}(x)$ is lsc on $\mathbb{R}^{N} \times[0, T]$, by first considering $A=\{(x, t) \in$ $\left.\mathbb{R}^{N} \times[0, T] ; x \notin K(t)\right\}$, which is open in $\mathbb{R}^{N} \times[0, T]$ by assumption. Let $\left(x_{n}, t_{n}\right) \in A$ converge to $(x, t) \in A$. Since $K(t)$ is closed and non-empty for all $t$, the distance of $x_{n}$ (resp. $\left.x\right)$ to $K\left(t_{n}\right)$ (resp. $K(t))$ is attained by a certain $y_{n}$ (resp. $y$ ):

$$
\left\{\begin{array} { l } 
{ d _ { K ( t _ { n } ) } ( x _ { n } ) = | x _ { n } - y _ { n } | , } \\
{ y _ { n } \in K ( t _ { n } ) , }
\end{array} \quad \left\{\begin{array}{l}
d_{K(t)}(x)=|x-y|, \\
y \in K(t) .
\end{array}\right.\right.
$$

Let us first consider the case of a finite accumulation point of $\left(d_{K\left(t_{n}\right)}\left(x_{n}\right)\right)$, given by the limit of $\left(\left|x_{n_{k}}-y_{n_{k}}\right|\right)$. The sequences $\left(x_{n_{k}}\right)$ and $\left(\left|x_{n_{k}}-y_{n_{k}}\right|\right)$ converge, thus $\left(y_{n_{k}}\right)$ is bounded. Passing to a subsequence if necessary, we can assume that it converges, say to $y_{\infty}$. Thus, since $u:(x, t) \mapsto$ $\mathbf{1}_{K(t)}(x)$ is usc, $u\left(y_{\infty}, t\right) \geqslant \lim \sup \left(u\left(y_{n_{k}}, t_{n_{k}}\right)\right)=1$, which means that $y_{\infty} \in K(t)$ and by definition of $y,|x-y| \leqslant\left|x-y_{\infty}\right|$, i.e. $d_{K(t)}(x) \leqslant \lim d_{K\left(t_{n_{k}}\right)}\left(x_{n_{k}}\right)$. This is also true for an infinite accumulation point, obviously. Finally, we remark that this also holds for a couple $(x, t)$ such that $x \in K(t)$, since in this case $d_{K(t)}(x)=0$, the minimum possible value of the distance function.

2. Let us now prove the second part of the assertion: for all $x \in \mathbb{R}^{N}, t \mapsto d_{K(t)}(x)$ is left continuous on $(0, T)$. Let us fix $t \in(0, T), \varepsilon>0$ and a sequence $\left(t_{n}\right)$ converging to $t$ from the left. Thanks to Proposition 2.3. $K(t) \subset K\left(t_{n}\right)+\bar{B}\left(0, L\left(t-t_{n}\right)\right) \subset K\left(t_{n}\right)+\bar{B}(0, \varepsilon)$ for $t_{n}$ close enough to $t$. Let $y \in K(t)$ be so that $d_{K(t)}(x)=|x-y|$. Then for $n$ large enough, $y \in K\left(t_{n}\right)+\bar{B}(0, \varepsilon)$, so that $d_{K\left(t_{n}\right)}(x) \leqslant d_{K(t)}(x)+\varepsilon$, and this proves that $t \mapsto d_{K(t)}(x)$ is left usc.

\subsection{Semiconvex functions and subdifferential of a convex function}

We refer to [12] for the notion of semiconvex function. Recall that a function $f: \Omega \rightarrow \mathbb{R}$ defined on a convex subset $\Omega$ of $\mathbb{R}^{N}$ is semiconvex with constant $M \geqslant 0$ if $x \mapsto f(x)+M|x|^{2} / 2$ is convex on $\Omega$ (and semiconcave if $-f$ semiconvex), which amounts to saying that for all $(x, y) \in \Omega^{2}$ and all $\lambda \in[0,1]$,

$$
f(\lambda x+(1-\lambda) y) \leqslant \lambda f(x)+(1-\lambda) f(y)+\frac{M}{2} \lambda(1-\lambda)|x-y|^{2} .
$$

For $f$ of class $C^{2}$ and $\Omega$ open, this is also equivalent to saying that for all $x \in \Omega, D^{2} f(x) \geqslant-M$ Id in the sense of symmetric matrices.

We will mainly use the notion of semiconvexity for the distance function to the front. The following lemma will be helpful: 
LEMmA 2.5 ([12, Proposition 2.2.2]) Let $F \neq \emptyset$ be a closed subsets of $\mathbb{R}^{N}$. For all convex subsets $\Omega$ such that $\gamma=\inf _{x \in \Omega} d_{F}(x)>0$, the distance function to $F, d_{F}$, is semiconcave on $\Omega$ with constant $1 / \gamma$.

For the notion of subdifferential of a convex function, we refer to [20].

DEFinition 2.6 Let $\Omega$ be a convex open subset of $\mathbb{R}^{N}$ and $f: \Omega \rightarrow \mathbb{R}$ be a convex function. The subdifferential of $f$ at $x \in \Omega$ is the set

$$
\partial_{*} f(x)=\left\{p \in \mathbb{R}^{N} ; \forall y \in \Omega, f(y) \geqslant f(x)+\langle p, y-x\rangle\right\} .
$$

If $f$ is concave, the superdifferential of $f$ at $x \in \Omega$ is the set

$$
\partial^{*} f(x)=\left\{p \in \mathbb{R}^{N} ; \forall y \in \Omega, f(y) \leqslant f(x)+\langle p, y-x\rangle\right\} .
$$

These sets are never empty thanks to the separation theorem. If $f$ is convex and differentiable at $x$, then $\partial_{*} f(x)=\{D f(x)\}$. The following lemma is straightforward but useful:

LEMMA 2.7 ([20]) Let $f_{n}, f$ be convex functions on a convex set $\Omega$ satisfying:

(i) For all sequences $y_{n} \rightarrow y$, limsup $f_{n}\left(y_{n}\right) \leqslant f(y)$.

(ii) There exists a sequence $x_{n} \rightarrow x$ such that $\lim f_{n}\left(x_{n}\right)=f(x)$.

(iii) $p_{n} \in \partial_{*} f_{n}\left(x_{n}\right)$ and $p_{n} \rightarrow p$.

Then $p \in \partial_{*} f(x)$.

\section{$2.3 \quad B V$ functions and sets of finite perimeter}

The results of this section are entirely taken from [15] unless explicitly stated otherwise. Let $\Omega$ be an open subset of $\mathbb{R}^{N}$.

\subsubsection{Functions of bounded variation}

DEFINITION 2.8 A function $f \in L_{\text {loc }}^{1}(\Omega)$ is said to have locally bounded variation in $\Omega$ if for all open subsets $U \subset \subset \Omega$ relatively compact in $\Omega$,

$$
\sup \left\{\int_{U} f(x) \operatorname{div} \phi(x) \mathrm{d} x ; \phi \in C_{c}^{1}\left(U, \mathbb{R}^{N}\right),\|\phi\|_{\infty} \leqslant 1\right\}<\infty .
$$

We denote by $B V_{\text {loc }}(\Omega)$ the set of functions of locally bounded variation in $\Omega$. We also say that $f \in L^{1}(\Omega)$ has bounded variation in $\Omega$ if the above condition holds for $U=\Omega$. We denote by $B V(\Omega)$ the set of functions of bounded variation in $\Omega$.

The Riesz representation theorem then yields:

THEOREM 2.9 Let $f \in B V_{\text {loc }}(\Omega)$. Then there exists a Radon measure $\mu$ on $\Omega$ and a $\mu$-measurable function $\sigma: \Omega \rightarrow \mathbb{R}^{N}$ such that:

1. $|\sigma(x)|=1 \mu$-a.e.

2. $\int_{\Omega} f(x) \operatorname{div} \phi(x) \mathrm{d} x=-\int_{\Omega}\langle\phi(x), \sigma(x)\rangle \mathrm{d} \mu \forall \phi \in C_{c}^{1}\left(\Omega, \mathbb{R}^{N}\right)$.

The measure $\mu$ is called the variation measure of $f$, denoted by $\|D f\|$, and we set $[D f]=\sigma\|D f\|$. We also have, for all open subsets $U \subset \subset \Omega$ relatively compact in $\Omega$,

$$
\|D f\|(U)=\sup \left\{\int_{U} f(x) \operatorname{div} \phi(x) \mathrm{d} x ; \phi \in C_{c}^{1}\left(U, \mathbb{R}^{N}\right),\|\phi\|_{\infty} \leqslant 1\right\} .
$$




\subsubsection{Sets of finite perimeter}

DEFinition 2.10 An $\mathcal{L}^{N}$-measurable subset $E \subset \mathbb{R}^{N}$ is said to have (locally) finite perimeter in $\Omega$ if $\mathbf{1}_{E}$ has (locally) bounded variation in $\Omega$. The variation measure of $\mathbf{1}_{E}$ in $\Omega$ is in this case denoted by $\|\partial E\|$, and the function $-\sigma$ given by Theorem 2.9 is denoted by $v_{E}$.

Definition 2.11 Let $E \subset \mathbb{R}^{N}$ be a set of locally finite perimeter in $\Omega$. We say that $x \in \Omega$ belongs to the reduced boundary of $E, \partial^{*} E$, if:

1. $\|\partial E\|(B(x, r))>0$ for all $r>0$ such that $B(x, r) \subset \Omega$,

2. $\frac{1}{\|\partial E\|(B(x, r))} \int_{B(x, r)} v_{E}(y) \mathrm{d}\|\partial E\| \underset{r \rightarrow 0}{\longrightarrow} v_{E}(x)$,

3. $\left|v_{E}(x)\right|=1$.

Then we have the following theorem:

THEOREM 2.12 (Gauss-Green formula) Let $E \subset \mathbb{R}^{N}$ be a set of locally finite perimeter in $\Omega$. Then for all $\phi \in C_{c}^{1}\left(\Omega, \mathbb{R}^{N}\right)$,

$$
\int_{E} \operatorname{div} \phi(x) \mathrm{d} x=\int_{\partial^{*} E}\left\langle\phi(x), v_{E}(x)\right\rangle \mathrm{d} \mathcal{H}^{N-1}(x) .
$$

Moreover we have control on the perimeter of level sets of the distance function to a closed non-empty set, as a consequence of the following proposition (see [1, Lemma 2.4]):

PROPOSITION 2.13 For all $r_{0}, r_{1}>0$ and $R>0$, there exists $M>0$ such that for all closed non-empty $E \subset \mathbb{R}^{N}$ with diameter smaller than $R$, and for all $r_{0} \leqslant r \leqslant r_{1}$, the set $E^{r}=\left\{x \in \mathbb{R}^{N}\right.$; $d_{E}(x)=r$ satisfies

$$
\mathcal{H}^{N-1}\left(E^{r}\right) \leqslant M .
$$

\section{An integral formulation of the eikonal equation for subsolutions}

We first focus on the notion of subsolution. All results can be adapted to supersolutions. The corresponding results and changes in the proofs are given in Section 6.

Let $c: \mathbb{R}^{N} \times[0, T] \rightarrow \mathbb{R}$ satisfy the assumptions of Theorem 2.2. and let $K:[0, T] \rightarrow$ $\mathcal{P}\left(\mathbb{R}^{N}\right) \backslash\{\emptyset\}$ be such that

1. $K(0)$ is compact, $K(t) \rightarrow K(0)$ as $t \rightarrow 0$ and $K(t) \rightarrow K(T)$ as $t \rightarrow T$ in the Hausdorff distance.

2. The graph $\bigcup_{t \in[0, T]}\{t\} \times K(t)$ of $K$ is closed in $[0, T] \times \mathbb{R}^{N}$.

3. $u:(x, t) \mapsto \mathbf{1}_{K(t)}(x)$ is a viscosity subsolution of the eikonal equation:

$$
u_{t}=c(x, t)|D u| \quad \text { in } \mathbb{R}^{N} \times(0, T) .
$$

Set, for all $\varepsilon>0$,

$$
K^{\varepsilon}(t)=\left\{x \in \mathbb{R}^{N} ; d_{K(t)}(x)<\varepsilon\right\}, \quad c^{\varepsilon}(x, t)=\max _{|y-x| \leqslant \varepsilon} c(y, t) .
$$

The aim of this section is the proof of the following result: 
THEOREM 3.1 (Integral formulation for subsolutions) For all $t_{1}$ and $t_{2}$ satisfying $0 \leqslant t_{1} \leqslant t_{2} \leqslant T$, for almost all $\varepsilon>0$, and for all $\phi \in C^{1}\left(\mathbb{R}^{N} \times[0, T], \mathbb{R}_{+}\right)$,

$$
\begin{aligned}
& \int_{t_{1}}^{t_{2}} \int_{K^{\varepsilon}(t)} \phi_{t}(x, t) \mathrm{d} x \mathrm{~d} t+\int_{t_{1}}^{t_{2}} \int_{\left\{d_{K(t)}=\varepsilon\right\}} c^{\varepsilon}(x, t) \phi(x, t) \mathrm{d} \mathcal{H}^{N-1}(x) \mathrm{d} t \\
& \geqslant\left[\int_{K^{\varepsilon}(t)} \phi(x, t) \mathrm{d} x\right]_{t_{1}}^{t_{2}} .
\end{aligned}
$$

To this end, we set $w(x, t)=-d_{K(t)}(x)$ so that for all $\varepsilon>0$,

$$
K^{\varepsilon}(t)=\left\{x \in \mathbb{R}^{N} ; w(x, t)>-\varepsilon\right\} .
$$

Let us fix $\varepsilon>0$ and $\theta: \mathbb{R} \rightarrow \mathbb{R}$ satisfying the following conditions:

1. $\theta$ is non-decreasing and smooth,

2. $\theta(x)=0$ if $x \leqslant-\varepsilon, \theta(x)=1$ if $x \geqslant 0$.

Set $w_{\theta}=\theta \circ w$. We start by giving a semiconvexity property of $w_{\theta}$ :

LEMmA 3.2 For all $(x, t) \in \mathbb{R}^{N} \times(0, T)$ such that $x \notin K(t)$, there exist $M>0, \delta>0$ and $r>0$ such that for all $s \in(t-\delta, t+\delta), w_{\theta}(\cdot, s)$ is semiconvex with constant $M$ on $B(x, r)$.

REMARK 3.3 In this lemma, the key point is local uniformity with respect to $s$ of the semiconvexity constant $M$.

Proof. Since $x \notin K(t)$, the lower semicontinuity of $(x, t) \mapsto d_{K(t)}(x)$ given by Proposition 2.4 implies that there exist $\gamma>0, \delta>0$ and $r>0$ such that $d_{K(s)}(y) \geqslant \gamma$ for every $s \in(t-\delta, t+\delta)$ and $y \in B(x, r)$. But then thanks to Lemma 2.5, $y \mapsto d_{K(s)}(y)$ is semiconcave on $B(x, r)$ with constant $1 / \gamma$. The conclusion now follows from [12, Proposition 2.1.12] on the semiconvexity of the composite of a smooth function with a semiconvex function.

We are now ready to begin the proof of Theorem 3.1 .

\subsection{Equation satisfied by $w_{\theta}$ in the viscosity sense}

This subsection closely follows the ideas of Soner [21] (see also [9, Theorem 3.1]). We provide the proof of the following proposition for sake of completeness.

Proposition $3.4 w_{\theta}:(x, t) \mapsto \theta\left(-d_{K(t)}(x)\right)$ is a subsolution of $\left(w_{\theta}\right)_{t}=c^{\varepsilon}(x, t)\left|D w_{\theta}\right|$ in $\mathbb{R}^{N} \times(0, T)$.

Proof. According to Proposition 2.4 $w$ is usc on $\mathbb{R}^{N} \times[0, T]$. Since $\theta$ is continuous and nondecreasing, $w_{\theta}$ is also usc.

Step 1. Let $\phi$ be of class $C^{1}$ and such that $w_{\theta}-\phi$ attains a local maximum at $\left(x_{0}, t_{0}\right) \in \mathbb{R}^{N} \times(0, T)$, which we can assume equal to 0, i.e. $w_{\theta}\left(x_{0}, t_{0}\right)=\phi\left(x_{0}, t_{0}\right)$. Let $y_{0}$ be such that $y_{0} \in K\left(t_{0}\right)$ and $w\left(x_{0}, t_{0}\right)=-\left|x_{0}-y_{0}\right|$ (if $x_{0} \in K\left(t_{0}\right)$, then $\left.y_{0}=x_{0}\right)$. Set $\psi(z, t)=\phi\left(z+x_{0}-y_{0}, t\right)$. Let us show that $u-\psi:(z, t) \mapsto \mathbf{1}_{K(t)}(z)-\psi(z, t)$ has a local maximum at $\left(y_{0}, t_{0}\right)$, which will enable us to use the fact that $u$ is a subsolution of 3.1. For $z$ close to $y_{0}$, and $t$ close to $t_{0}$,

$$
-\psi(z, t)=-\phi\left(z+x_{0}-y_{0}, t\right) \leqslant-w_{\theta}\left(z+x_{0}-y_{0}, t\right)
$$


because $w_{\theta}-\phi$ has a local maximum at $\left(x_{0}, t_{0}\right)$ where it vanishes. Since by definition we have $w\left(z+x_{0}-y_{0}, t\right)=-d_{K(t)}\left(z+x_{0}-y_{0}\right)$, for $z \in K(t)$ we get

$$
-\psi(z, t) \leqslant-\theta\left(-d_{K(t)}\left(z+x_{0}-y_{0}\right)\right) \leqslant-\theta\left(-\left|x_{0}-y_{0}\right|\right)
$$

by definition of $d_{K(t)}$, and because $\theta$ is non-decreasing. But

$$
-\theta\left(-\left|x_{0}-y_{0}\right|\right)=-\theta\left(w\left(x_{0}, t_{0}\right)\right)=-\phi\left(x_{0}, t_{0}\right)=-\psi\left(y_{0}, t_{0}\right)
$$

and $\mathbf{1}_{K(t)}(z)=\mathbf{1}_{K\left(t_{0}\right)}\left(y_{0}\right)=1$, so $u(z, t)-\psi(z, t) \leqslant u\left(y_{0}, t_{0}\right)-\psi\left(y_{0}, t_{0}\right)$. Moreover, $-\psi(z, t) \rightarrow$ $-\psi\left(y_{0}, t_{0}\right)$ as $(z, t) \rightarrow\left(y_{0}, t_{0}\right)$, thus in a neighborhood of $\left(y_{0}, t_{0}\right)$,

$$
-\psi(z, t) \leqslant 1-\psi\left(y_{0}, t_{0}\right) .
$$

Therefore if $z \notin K(t)$, then $\mathbf{1}_{K(t)}(z)=0, \mathbf{1}_{K\left(t_{0}\right)}\left(y_{0}\right)=1$, and 3.3$)$ means that, again, $u(z, t)-$ $\psi(z, t) \leqslant u\left(y_{0}, t_{0}\right)-\psi\left(y_{0}, t_{0}\right)$, which is the desired result.

Step 2. Since $u$ is a subsolution of 3.1 , according to Step 1 we have $\psi_{t}\left(y_{0}, t_{0}\right) \leqslant$ $c\left(y_{0}, t_{0}\right)\left|D \psi\left(y_{0}, t_{0}\right)\right|$, that is,

$$
\phi_{t}\left(x_{0}, t_{0}\right) \leqslant c\left(y_{0}, t_{0}\right)\left|D \phi\left(x_{0}, t_{0}\right)\right| .
$$

This is where we see the interest of perturbing the equation with $c^{\varepsilon}$ and of truncating with $\theta$, because if $d_{K\left(t_{0}\right)}\left(x_{0}\right)=\left|x_{0}-y_{0}\right| \leqslant \varepsilon$, then $c\left(y_{0}, t_{0}\right) \leqslant c^{\varepsilon}\left(x_{0}, t_{0}\right)$, and therefore $\phi_{t}\left(x_{0}, t_{0}\right) \leqslant$ $c^{\varepsilon}\left(x_{0}, t_{0}\right)\left|D \phi\left(x_{0}, t_{0}\right)\right|$. Moreover if on the contrary $d_{K\left(t_{0}\right)}\left(x_{0}\right)=-w\left(x_{0}, t_{0}\right)=\left|x_{0}-y_{0}\right|>\varepsilon$, then since $w$ is usc, it follows that $w(x, t)<-\varepsilon$ in a neighborhood of $\left(x_{0}, t_{0}\right)$, thus $w_{\theta}(x, t)=0$ locally and the equation is still satisfied.

\subsection{Variational equation satisfied by $w_{\theta}$}

Proposition 3.5 For all test functions $\phi \in C_{c}^{1}\left(\mathbb{R}^{N} \times(0, T), \mathbb{R}_{+}\right)$,

$$
\int_{0}^{T} \int_{\mathbb{R}^{N}} w_{\theta}(x, t) \phi_{t}(x, t) \mathrm{d} x \mathrm{~d} t+\int_{0}^{T} \int_{\mathbb{R}^{N}} c^{\varepsilon}(x, t)\left|D w_{\theta}(x, t)\right| \phi(x, t) \mathrm{d} x \mathrm{~d} t \geqslant 0 .
$$

Proof. In order to prove this proposition, we apply a technique of regularization of $w_{\theta}$ in time by sup-convolution: we define for $\sigma>0$,

$$
w_{\theta}^{\sigma}(x, t)=\max _{(y, s) \in \mathbb{R}^{N} \times[0, T]}\left\{w_{\theta}(y, s)-\frac{1}{\sigma}\left[|x-y|^{2}+(t-s)^{2}\right]\right\} .
$$

This is justified: $w_{\theta}$ is usc, so $(y, s) \mapsto w_{\theta}(y, s)-\frac{1}{\sigma}\left[|x-y|^{2}+(t-s)^{2}\right]$ is also usc. In addition, $w_{\theta}$ is bounded, so

$$
(y, s) \mapsto w_{\theta}(y, s)-\frac{1}{\sigma}\left[|x-y|^{2}+(t-s)^{2}\right]
$$

is coercive. Consequently, the supremum is indeed a maximum. Moreover it is known that 3.5 defines a locally Lipschitz continuous function on $\mathbb{R}^{N} \times[0, T]$ (while $w_{\theta}$ was only Lipschitz continuous with respect to the space variable), converging pointwise to $w_{\theta}$ as $\sigma$ converges to 0 . 
Step 1. Let us show, in the spirit of Step 1 of the previous proof, that $w_{\theta}^{\sigma}$ is a viscosity subsolution of $\left(w_{\theta}^{\sigma}\right)_{t}=\left[c^{\varepsilon}\right]^{h(\sigma)}(x, t)\left|D w_{\theta}^{\sigma}\right|$ in $\mathbb{R}^{N} \times(h(\sigma), T-h(\sigma))$, where $h(\sigma) \in(0,1]$ tends to 0 as $\sigma \rightarrow 0$, and where we have set

$$
\left[c^{\varepsilon}\right]^{\alpha}(x, t)=\max _{|(y, s)-(x, t)| \leqslant \alpha} c^{\varepsilon}(y, s) .
$$

Let $\left(x_{0}, t_{0}\right) \in \mathbb{R}^{N} \times(0, T)$. There exists $\left(y_{0}, s_{0}\right) \in \mathbb{R}^{N} \times[0, T]$ such that $w_{\theta}^{\sigma}\left(x_{0}, t_{0}\right)=w_{\theta}\left(y_{0}, s_{0}\right)-$ $\frac{1}{\sigma}\left[\left|x_{0}-y_{0}\right|^{2}+\left(t_{0}-s_{0}\right)^{2}\right]$. We set $\mathbf{u}=\left(x_{0}, t_{0}\right), \mathbf{v}=\left(y_{0}, s_{0}\right)$ for the sake of readability. Then

$$
0 \leqslant w_{\theta}^{\sigma}(\mathbf{u})=w_{\theta}(\mathbf{v})-\frac{1}{\sigma}|\mathbf{u}-\mathbf{v}|^{2} \leqslant 1-\frac{1}{\sigma}|\mathbf{u}-\mathbf{v}|^{2}
$$

so $|\mathbf{u}-\mathbf{v}| \leqslant \sigma^{1 / 2}=: h(\sigma)$ and hence $\mathbf{v} \in \mathbb{R}^{N} \times(0, T)$ for $\mathbf{u} \in \mathbb{R}^{N} \times(h(\sigma), T-h(\sigma))$. Therefore we can use the equation satisfied by $w_{\theta}$ in $\mathbb{R}^{N} \times(0, T)$ to deduce the equation satisfied by $w_{\theta}^{\sigma}$ in $\mathbb{R}^{N} \times(h(\sigma), T-h(\sigma))$, as in [18].

Step 2. Let $\phi \in C_{c}^{1}\left(\mathbb{R}^{N} \times(0, T), \mathbb{R}_{+}\right)$, and $U$ be an open and relatively compact subset of $\mathbb{R}^{N} \times$ $(0, T)$ such that $\phi$ vanishes on $U^{c}$. We can choose $\sigma$ so small that $U \subset \mathbb{R}^{N} \times(h(\sigma), T-h(\sigma))$, and then $w_{\theta}^{\sigma}$ is a viscosity subsolution of $\left(w_{\theta}^{\sigma}\right)_{t}=\left[c^{\varepsilon}\right]^{h(\sigma)}(x, t)\left|D w_{\theta}^{\sigma}\right|$ in $U$ thanks to Step 1 .

Since $w_{\theta}^{\sigma}$ is locally Lipschitz continuous, it is differentiable almost everywhere and the inequality $\left(w_{\theta}^{\sigma}\right)_{t} \leqslant\left[c^{\varepsilon}\right]^{h(\sigma)}(x, t)\left|D w_{\theta}^{\sigma}\right|$ actually holds a.e. in $U$ (see [7]). Therefore,

$$
\int_{\mathbb{R}^{N} \times(0, T)}\left[\left(w_{\theta}^{\sigma}\right)_{t}-\left[c^{\varepsilon}\right]^{h(\sigma)}(x, t)\left|D w_{\theta}^{\sigma}\right|\right] \phi \leqslant 0,
$$

this integral being in fact taken on $U$. But $w_{\theta}^{\sigma}$ is locally Lipschitz continuous and therefore belongs to $W_{\text {loc }}^{1, \infty}$, and its a.e. time derivative coincides with its time derivative in the sense of distributions. Thus

$$
\int_{\mathbb{R}^{N} \times(0, T)}\left[w_{\theta}^{\sigma} \phi_{t}+\left[c^{\varepsilon}\right]^{h(\sigma)}(x, t)\left|D w_{\theta}^{\sigma}\right| \phi\right] \geqslant 0 .
$$

Now we want to pass to the limit in this expression as $\sigma \rightarrow 0$ by applying the dominated convergence theorem.

Step 3. To do so we notice that $w_{\theta}^{\sigma} \phi_{t}$ and $\left[c^{\varepsilon}\right]^{h(\sigma)} \phi$ are bounded on $U$ uniformly in $\sigma$ since $\left|w_{\theta}^{\sigma}\right| \leqslant 1$ and $c$ is bounded. Moreover $w_{\theta}^{\sigma}$ converges pointwise to $w_{\theta}$ as $\sigma \rightarrow 0$ as we recalled above, and $\left[c^{\varepsilon}\right]^{h(\sigma)}$ converges pointwise to $c^{\varepsilon}$. It only remains to deal with the term $\left|D w_{\theta}^{\sigma}\right|$.

To this end, we fix a sequence $\left(\sigma_{n}\right)$ converging to $0^{+}$. There exists a subset $\hat{U} \subset U$ such that $\mathcal{L}^{N}(U \backslash \hat{U})=0$ and $D w_{\theta}, D w_{\theta}^{\sigma_{n}}$ are defined on $\hat{U}$ for all $n$. Let us show that $\left(D w_{\theta}^{\sigma_{n}}\right)$ converges a.e. to $D w_{\theta}$ on $\hat{U}$ as $n \rightarrow \infty$ :

Lemma 3.6 For almost all $(x, t) \in \hat{U}, D w_{\theta}^{\sigma_{n}}(x, t) \rightarrow D w_{\theta}(x, t)$ as $n \rightarrow \infty$.

Proof. First case: $x \notin K(t)$. Let us fix, thanks to Lemma 3.2, $M>0, \delta>0$ and $r>0$ such that for all $s \in(t-\delta, t+\delta), w_{\theta}(\cdot, s)$ is semiconvex with constant $M$ on $B(x, r)$. For all $n$, choose $\left(y_{n}, s_{n}\right) \in \mathbb{R}^{N} \times(0, T)$ realizing the supremum: $w_{\theta}^{\sigma_{n}}(x, t)=w_{\theta}\left(y_{n}, s_{n}\right)-\frac{1}{\sigma_{n}}\left[\left|x-y_{n}\right|^{2}+\left(t-s_{n}\right)^{2}\right]$. Recall that $\left|(x, t)-\left(y_{n}, s_{n}\right)\right| \leqslant h\left(\sigma_{n}\right) \rightarrow 0$, so that we can assume that for all $n$,

$$
\psi: y \mapsto w_{\theta}(y, t)+\frac{M}{2}|y|^{2} \quad \text { and } \quad \psi_{n}: y \mapsto w_{\theta}\left(y, s_{n}\right)+\frac{M}{2}|y|^{2}
$$

are convex on $B(x, r)$, and that $y_{n} \in B(x, r)$. 
We know, thanks to Corollary 10.14 of [20], that $D w_{\theta}^{\sigma_{n}}(x, t)=\frac{2}{\sigma_{n}}\left(y_{n}-x\right)$. Let us compute $D w_{\theta}\left(y_{n}, s_{n}\right)$ : for $z$ close to $y_{n}$,

$$
w_{\theta}\left(z, s_{n}\right)-\frac{1}{\sigma_{n}}\left[|x-z|^{2}+\left(t-s_{n}\right)^{2}\right] \leqslant w_{\theta}\left(y_{n}, s_{n}\right)-\frac{1}{\sigma_{n}}\left[\left|x-y_{n}\right|^{2}+\left(t-s_{n}\right)^{2}\right],
$$

so

$$
w_{\theta}\left(z, s_{n}\right) \leqslant w_{\theta}\left(y_{n}, s_{n}\right)+\frac{1}{\sigma_{n}}\left\{|x-z|^{2}-\left|x-y_{n}\right|^{2}\right\} .
$$

But $|x-z|^{2}-\left|x-y_{n}\right|^{2}=\left|z-y_{n}\right|^{2}+2\left\langle z-y_{n}, y_{n}-x\right\rangle$, so we obtain

$$
w_{\theta}\left(z, s_{n}\right) \leqslant w_{\theta}\left(y_{n}, s_{n}\right)+\frac{2}{\sigma_{n}}\left\langle z-y_{n}, y_{n}-x\right\rangle+\frac{1}{\sigma_{n}}\left|z-y_{n}\right|^{2} .
$$

Therefore $\frac{2}{\sigma_{n}}\left(y_{n}-x\right)$ is a Fréchet superdifferential (see [12]) for $w_{\theta}\left(\cdot, s_{n}\right)$ at $y_{n}$. But, in addition, $w_{\theta}\left(\cdot, s_{n}\right)$ is semiconvex on $B(x, r) \ni y_{n}$, so differentiable at $y_{n}$, and its gradient equals $\frac{2}{\sigma_{n}}\left(y_{n}-x\right)$ (see [12, Proposition 3.1.5]). To sum up, for all $n$ we have $D w_{\theta}^{\sigma_{n}}(x, t)=D w_{\theta}\left(y_{n}, s_{n}\right)=: p_{n}$.

Since $s_{n} \rightarrow t$ and $w_{\theta}$ is usc, for all sequences $z_{n} \rightarrow z$ we have $\lim \sup \psi_{n}\left(z_{n}\right) \leqslant \psi(z)$, where $\psi_{n}$ and $\psi$ are defined by (3.7). Moreover $y_{n} \rightarrow x$ and since $w_{\theta}^{\sigma_{n}}$ converges pointwise to $w_{\theta}$, we have

$$
\psi_{n}\left(y_{n}\right)=w_{\theta}^{\sigma_{n}}\left(y_{n}, t\right)+\frac{M}{2}\left|y_{n}\right|^{2} \underset{n \rightarrow \infty}{\longrightarrow} w_{\theta}(x, t)+\frac{M}{2}|x|^{2}=\psi(x) .
$$

But $p_{n}=D w_{\theta}^{\sigma_{n}}(x, t)=D w_{\theta}\left(y_{n}, s_{n}\right)$, so $\left(p_{n}\right)$ is bounded: for all $s, w_{\theta}(s, \cdot)$ is $\left\|\theta^{\prime}\right\|_{\infty}$-Lipschitz continuous, so $\left\|D w_{\theta}\right\|_{\infty} \leqslant\left\|\theta^{\prime}\right\|_{\infty}$, and the same holds for $D w_{\theta}^{\sigma_{n}}$ independently of $n$. We can extract a subsequence $\left(n_{k}\right)$ such that $\left(p_{n_{k}}\right)$ converges to some $p \in \mathbb{R}^{N}$. Then $D \psi_{n_{k}}\left(y_{n_{k}}\right)=p_{n_{k}}+M y_{n_{k}} \rightarrow$ $p+M x$. As a consequence, thanks to Lemma 2.7, $p+M x \in \partial_{*} \psi(x)$. But $w_{\theta}$ is differentiable at $(x, t)$ with respect to $x$, so $\partial_{*} \psi(x)$ reduces to its gradient $D w_{\theta}(x, t)+M x$, which shows that $p+M x=D w_{\theta}(x, t)+M x$, and $p=D w_{\theta}(x, t)$. This holds for all converging subsequences of $\left(p_{n}\right)$, so $p_{n} \rightarrow D w_{\theta}(x, t)$, that is, $D w_{\theta}^{\sigma_{n}}(x, t) \rightarrow D w_{\theta}(x, t)$ as $n \rightarrow \infty$.

Second case: $x \in K(t)$. Since $w_{\theta}^{\sigma_{n}} \geqslant w_{\theta}$ and $w_{\theta}^{\sigma_{n}} \leqslant \max w_{\theta}=1$, for all $n$ we have $w_{\theta}^{\sigma_{n}}(x, t)=$ $w_{\theta}(x, t)=1$. A result due to Stampacchia (see for instance [15]) asserts that $D w_{\theta}=D w_{\theta}^{\sigma_{n}}=0$ almost everywhere on $\left\{w_{\theta}=1\right\}$ since all $w_{\theta}^{\sigma_{n}}$ and $w_{\theta}$ are Lipschitz continuous with respect to the space variable. So $D w_{\theta}^{\sigma_{n}}(x, t) \rightarrow D w_{\theta}(x, t)$ as $n \rightarrow \infty$ for almost all $(x, t)$ such that $x \in K(t)$.

Finally, $D w_{\theta}^{\sigma}$ is bounded on $U$ uniformly in $\sigma$ : as we have seen above, $\left\|D w_{\theta}^{\sigma}\right\|_{\infty} \leqslant\left\|\theta^{\prime}\right\|_{\infty}$ for all $\sigma>0$. The use of the dominated convergence theorem is justified and proves Proposition 3.5

\subsection{The integral formulation}

We are now ready to conclude the proof of Theorem 3.1 Fix $0<\alpha_{1}<\alpha_{2}<\varepsilon$.

Step 1. We first notice that an approximation argument shows that 3.4 also holds for the following function $\theta$, although it is not of class $C^{1}$ :

1. $\theta$ is non-decreasing and continuous,

2. $\theta(x)=0$ if $x \leqslant-\alpha_{2}, \theta(x)=1$ if $x \geqslant-\alpha_{1}$,

3. $\theta$ is affine on $\left[-\alpha_{2},-\alpha_{1}\right]$. 
Now we would like to transform the second term of (3.4) so as to get rid of $\left|D w_{\theta}\right|$. To do so we interpret it as a jacobian thanks to the co-area formula (see [15]), which yields, for all $\phi \in$ $C_{c}^{1}\left(\mathbb{R}^{N} \times(0, T), \mathbb{R}_{+}\right)$

$\int_{0}^{T} \int_{\mathbb{R}^{N}} w_{\theta}(x, t) \phi_{t}(x, t) \mathrm{d} x \mathrm{~d} t+\int_{0}^{T} \int_{0}^{1} \int_{\left\{w_{\theta}(\cdot, t)=\tau\right\}} c^{\varepsilon}(x, t) \phi(x, t) \mathrm{d} \mathcal{H}^{N-1}(x) \mathrm{d} \tau \mathrm{d} t \geqslant 0$,

where the integral from 0 to 1 represents the values taken by $w_{\theta}(\cdot, t)$.

Step 2. For $\tau \in(0,1), w_{\theta}(x, t)=\tau \Leftrightarrow w(x, t)=\theta^{-1}(\tau) \Leftrightarrow d_{K(t)}(x)=-\theta^{-1}(\tau) \in\left(\alpha_{1}, \alpha_{2}\right)$. Moreover, Proposition 2.3 shows that the diameter of $K(t)$ is uniformly bounded on [0,T]. Therefore Proposition 2.13 guarantees the existence of a constant $M>0$ such that for all $\tau \in(0,1)$,

$$
\mathcal{H}^{N-1}\left(\left\{w_{\theta}(\cdot, t)=\tau\right\}\right) \leqslant M .
$$

Step 3. Let us now transform each of the terms of (3.8) so as to get rid of $w_{\theta}$. For the first term, we simply notice that

$$
\int_{0}^{T} \int_{\mathbb{R}^{N}} w_{\theta}(x, t) \phi_{t}(x, t) \mathrm{d} x \mathrm{~d} t=\int_{0}^{T} \int_{\left\{w(\cdot, t)>-\alpha_{2}\right\}} w_{\theta}(x, t) \phi_{t}(x, t) \mathrm{d} x \mathrm{~d} t
$$

since $\theta(\sigma)=0$ if $\sigma \leqslant-\alpha_{2}$. In the second term, let us make the change of variable $\tau=\theta(-\sigma)$ for $\sigma \in\left(\alpha_{1}, \alpha_{2}\right)$ and $\tau \in(0,1)$. In this case $w_{\theta}(x, t)=\tau \Leftrightarrow \theta(w(x, t))=\theta(-\sigma) \Leftrightarrow w(x, t)=-\sigma$. Therefore we obtain, for all $\phi \in C_{c}^{1}\left(\mathbb{R}^{N} \times(0, T), \mathbb{R}_{+}\right)$and all $\alpha_{1}, \alpha_{2}$ satisfying $0<\alpha_{1}<\alpha_{2}<\varepsilon$,

$$
\begin{aligned}
\int_{0}^{T} \int_{\left\{w(\cdot, t)>-\alpha_{2}\right\}} & w_{\theta}(x, t) \phi_{t}(x, t) \mathrm{d} x \mathrm{~d} t \\
& +\frac{1}{\alpha_{2}-\alpha_{1}} \int_{\alpha_{1}}^{\alpha_{2}} \int_{0}^{T} \int_{\{w(\cdot, t)=-\sigma\}} c^{\varepsilon}(x, t) \phi(x, t) \mathrm{d} \mathcal{H}^{N-1}(x) \mathrm{d} t \mathrm{~d} \sigma \geqslant 0,
\end{aligned}
$$

where we have switched the integration order between $t$ and $\sigma$, which is permitted since for all $(t, \sigma)$ in the bounded domain $(0, T) \times\left(\alpha_{1}, \alpha_{2}\right)$,

$$
\left|\int_{\{w(\cdot, t)=-\sigma\}} c^{\varepsilon}(x, t) \phi(x, t) \mathrm{d} \mathcal{H}^{N-1}(x)\right| \leqslant M\|c\|_{\infty}\|\phi\|_{\infty}
$$

where $M$ denotes the constant given by (3.9).

Step 4. We now use the freedom in the choice of $\alpha_{1}$ and $\alpha_{2}$ to deduce from 3.10) a pointwise property of the integrand. To this end, we apply the Lebesgue-Besicovitch differentiation theorem (see for instance [15]) to the function

$$
\sigma \mapsto \int_{0}^{T} \int_{\{w(\cdot, t)=-\sigma\}} c^{\varepsilon}(x, t) \phi(x, t) \mathrm{d} \mathcal{H}^{N-1}(x) \mathrm{d} t,
$$

which lies in $L_{\text {loc }}^{1}(0, \varepsilon)$ for any $\phi \in C_{c}^{1}\left(\mathbb{R}^{N} \times(0, T), \mathbb{R}_{+}\right)$thanks to 3.11). Fixing $\sigma \in(0, \varepsilon)$ and choosing $\alpha_{1}=\sigma-\tau, \alpha_{2}=\sigma+\tau^{2}$ in (3.10) with $\tau \rightarrow 0$, we deduce, since $w_{\theta} \rightarrow \mathbf{1}_{K^{\sigma}(t)}$ for this choice of $\alpha_{1}$ and $\alpha_{2}$, that for almost all $\sigma \in(0, \varepsilon)$,

$$
\int_{0}^{T} \int_{\{w(\cdot, t)>-\sigma\}} \phi_{t}(x, t) \mathrm{d} x \mathrm{~d} t+\int_{0}^{T} \int_{\{w(\cdot, t)=-\sigma\}} c^{\varepsilon}(x, t) \phi(x, t) \mathrm{d} \mathcal{H}^{N-1}(x) \mathrm{d} t \geqslant 0 .
$$


Step 5. What we have done holds for a fixed $\phi \in C_{c}^{1}\left(\mathbb{R}^{N} \times(0, T), \mathbb{R}_{+}\right)$and $\varepsilon>0$. We now extend the result to all $\phi \in C_{c}^{1}\left(\mathbb{R}^{N} \times(0, T), \mathbb{R}_{+}\right)$and $\varepsilon>0$, using the fact that $C_{c}^{1}\left(\mathbb{R}^{N} \times(0, T), \mathbb{R}_{+}\right)$, equipped with the $C^{1}$ norm, and $\mathbb{R}$, are separable. This shows that $(3.12)$ holds for almost all $\sigma>0$, all $\varepsilon>\sigma$ and all $\phi \in C_{c}^{1}\left(\mathbb{R}^{N} \times(0, T), \mathbb{R}_{+}\right)$.

Let us fix such a $\sigma>0$. Letting $\varepsilon \rightarrow \sigma$ in (3.12) gives the integral formulation for test functions with compact support: since $\{w(\cdot, t)>-\varepsilon\}=K^{\varepsilon}(t)$ and $\{w(\cdot, t)=-\varepsilon\}=\left\{d_{K(t)}=\varepsilon\right\}$, we have for almost all $\varepsilon>0$, and all $\phi \in C_{c}^{1}\left(\mathbb{R}^{N} \times(0, T), \mathbb{R}_{+}\right)$,

$$
\int_{0}^{T} \int_{K^{\varepsilon}(t)} \phi_{t}(x, t) \mathrm{d} x \mathrm{~d} t+\int_{0}^{T} \int_{\left\{d_{K(t)}=\varepsilon\right\}} c^{\varepsilon}(x, t) \phi(x, t) \mathrm{d} \mathcal{H}^{N-1}(x) \mathrm{d} t \geqslant 0 .
$$

Step 6. To conclude, it remains to generalize (3.13) to test functions with no assumption on the support. Let $0<t_{1}<t_{2}<T$ and $\alpha$ be a smooth non-negative function equal to 1 on $\left[t_{1}, t_{2}\right]$ and having compact support in $(0, T)$. Let $\phi \in C^{1}\left(\mathbb{R}^{N} \times[0, T], \mathbb{R}_{+}\right)$with compact support in the space variable. Applying (3.13) to $\alpha \phi$ yields, for almost all $\varepsilon>0$,

$$
\begin{aligned}
& \int_{0}^{T} \int_{K^{\varepsilon}(t)} \alpha(t) \phi_{t}(x, t) \mathrm{d} x \mathrm{~d} t+\int_{0}^{T} \int_{K^{\varepsilon}(t)} \alpha_{t}(t) \phi(x, t) \mathrm{d} x \mathrm{~d} t \\
&+\int_{0}^{T} \int_{\left\{d_{K(t)=\varepsilon\}}\right.} c^{\varepsilon}(x, t) \alpha(t) \phi(x, t) \mathrm{d} \mathcal{H}^{N-1}(x) \mathrm{d} t \geqslant 0,
\end{aligned}
$$

which gives 3.2 when $\alpha$ converges to the indicator function of $\left[t_{1}, t_{2}\right]$ :

$\int_{t_{1}}^{t_{2}} \int_{K^{\varepsilon}(t)} \phi_{t}(x, t) \mathrm{d} x \mathrm{~d} t-\left[\int_{K^{\varepsilon}(t)} \phi(x, t) \mathrm{d} x\right]_{t_{1}}^{t_{2}}+\int_{t_{1}}^{t_{2}} \int_{\left\{d_{K(t)}=\varepsilon\right\}} c^{\varepsilon}(x, t) \phi(x, t) \mathrm{d} \mathcal{H}^{N-1}(x) \mathrm{d} t \geqslant 0$.

This holds for almost all $t_{1}$ and $t_{2}$ in $(0, T)$ and therefore for all $0<t_{1} \leqslant t_{2}<T$ since $\mathbf{1}_{K^{\varepsilon}(t)} \rightarrow$ $\mathbf{1}_{K^{\varepsilon}\left(t_{0}\right)}$ in $L^{1}\left(\mathbb{R}^{N}\right)$ as $t \rightarrow t_{0}^{-}$thanks to Propositions 2.4 and 2.13 (indeed, the latter guarantees that all sets of the form $\left\{d_{K\left(t_{0}\right)}=\varepsilon\right\}$ have zero $\mathcal{L}^{N}$ measure). This being also true for $t_{1} \rightarrow 0^{+}$ and $t_{2} \rightarrow T^{-}$by assumption 1 before Theorem 3.1, we see that 3.2 also holds for $t_{1}=0$ and $t_{2}=T$. Moreover, since the evolution is bounded, the time-dependent domain of integration $K^{\varepsilon}(t)$ is uniformly bounded. Thus if $\phi$ does not have compact support in the space variable either, after truncating $\phi$ in a $C^{1}$ way off a large ball if necessary, we see that 3.2$)$ holds for $\phi \in C^{1}\left(\mathbb{R}^{N} \times\right.$ $\left.[0, T], \mathbb{R}_{+}\right)$. This concludes the proof of Theorem 3.1

\section{Conversely: from the integral formulation to the notion of subsolution}

In this section we are interested in the converse of Theorem 3.1.

THEOREM 4.1 Let $c: \mathbb{R}^{N} \times[0, T] \rightarrow \mathbb{R}$ satisfy the assumptions of Theorem 2.2 and $K:[0, T] \rightarrow$ $\mathcal{P}\left(\mathbb{R}^{N}\right) \backslash\{\emptyset\}$ be such that

1. $K$ is uniformly bounded on $[0, T]$.

2. The graph $\bigcup_{t \in[0, T]}\{t\} \times K(t)$ of $K$ is closed in $[0, T] \times \mathbb{R}^{N}$.

3. Inequality (3.2) holds for all $0 \leqslant t_{1} \leqslant t_{2} \leqslant T$, for almost all $\varepsilon>0$ small enough and for all $\phi \in C^{1}\left(\mathbb{R}^{N} \times[0, T], \mathbb{R}_{+}\right)$.

Then $u:(x, t) \mapsto \mathbf{1}_{K(t)}(x)$ is a viscosity subsolution of $u_{t}=c(x, t)|D u|$ in $\mathbb{R}^{N} \times(0, T)$. 
During the proof of this theorem, we will need to use neighborhoods of a particular form and the corresponding notion of open sets:

Definition 4.2 A set of the form $B(x, r) \times(t-h, t]$ with $r>0, h>0$ is called a left neighborhood of $(x, t) \in \mathbb{R}^{N} \times(0, T)$. We say that $U \subset \mathbb{R}^{N} \times(0, T)$ is left open if $U$ contains a left neighborhood of each of its points.

We also define the corresponding notion of viscosity left subsolution, in which the test is restricted to left neighborhoods:

Definition 4.3 Let $U \subset \mathbb{R}^{N} \times(0, T)$ be left open. We say that $u$ is a viscosity left subsolution of $u_{t}=c(x, t)|D u|$ in $U$ if $u$ is usc on $\mathbb{R}^{N} \times[0, T]$ and if for all $(x, t) \in U$, for all test functions $\phi$ of class $C^{1}$ on $\mathbb{R}^{N} \times(0, T)$ such that $u-\phi$ has a maximum on a left neighborhood of $(x, t)$, we have $\phi_{t}(x, t) \leqslant c(x, t)|D \phi(x, t)|$.

It is easy to see that all classical results on viscosity solutions (stability, invariance, how a subsolution provides a set-theoretic subsolution) still hold for this notion of subsolution, under the only assumption in the stability theorem that the upper relaxed semilimit $\lim \sup ^{*}\left(u_{n}\right)$ of $\left(u_{n}\right)$ (see [7]) satisfies

$$
\lim \sup ^{*}\left(u_{n}\right)(x, t)=\lim \sup \left\{u_{n}\left(x_{n}, t_{n}\right) ; t_{n} \rightarrow t, t_{n} \leqslant t, x_{n} \rightarrow x\right\},
$$

i.e. we ask that the lim sup be achieved through lower times. We are now ready to begin the proof of Theorem 4.1 .

Proof of Theorem 4.4. The fact that $\bigcup_{t \in[0, T]}\{t\} \times K(t)$ is closed in $[0, T] \times \mathbb{R}^{N}$ ensures that $(x, t) \mapsto \mathbf{1}_{K(t)}(x)$ is usc. For the rest of the proof let us fix $\varepsilon_{0}$ such that 3.2$)$ holds for almost all $\varepsilon \in\left(0, \varepsilon_{0}\right)$.

Step 1. Let us first prove that for all $\left(x_{0}, t_{0}\right) \in \mathbb{R}^{N} \times(0, T)$ such that $x_{0} \in K^{\varepsilon_{0}}\left(t_{0}\right), t \mapsto d_{K(t)}\left(x_{0}\right)$ is left continuous at $t_{0}$. If this were not true, there would exist $t_{0} \in(0, T), x_{0} \in K^{\varepsilon_{0}}\left(t_{0}\right), \eta>0$ and a sequence $t_{n} \rightarrow t_{0}^{-}$such that for all $n \geqslant 1$,

$$
d_{K\left(t_{0}\right)}\left(x_{0}\right)+\eta<d_{K\left(t_{n}\right)}\left(x_{0}\right) .
$$

Let us choose $\alpha \in(0, \eta / 2)$ and $\phi \in C_{c}^{1}\left(B\left(x_{0}, \alpha\right), \mathbb{R}_{+}\right)$with $\phi\left(x_{0}\right)>0$. Assumption 3 implies that (3.2) holds with $\varepsilon=d_{K\left(t_{0}\right)}\left(x_{0}\right)+\delta$ for almost all $\delta \in(0, \eta / 2)$ small enough. Let us apply it to the time-independent function $(x, t) \mapsto \phi(x)$ between $t_{n}$ and $t_{0}$ for such a $\delta$ :

$$
\int_{t_{n}}^{t_{0}} \int_{\left\{d_{K(t)}=\varepsilon\right\}} c^{\varepsilon}(x, t) \phi(x) \mathrm{d} \mathcal{H}^{N-1}(x) \mathrm{d} t \geqslant\left[\int_{K^{\varepsilon}(t)} \phi(x) \mathrm{d} x\right]_{t_{n}}^{t_{0}} .
$$

But $\left[\int_{K^{\varepsilon}(t)} \phi(x) \mathrm{d} x\right]_{t_{n}}^{t_{0}}=\int_{K^{\varepsilon}\left(t_{0}\right)} \phi(x) \mathrm{d} x$ because (4.1) implies that $K^{\varepsilon}\left(t_{n}\right) \cap B\left(x_{0}, \alpha\right)=\emptyset$ for all $n \geqslant 1$. Moreover

$$
\int_{t_{n}}^{t_{0}} \int_{\left\{d_{K(t)}=\varepsilon\right\}} c^{\varepsilon}(x, t) \phi(x) \mathrm{d} \mathcal{H}^{N-1}(x) \mathrm{d} t \leqslant\left(t_{0}-t_{n}\right) M\|c\|_{\infty}\|\phi\|_{\infty},
$$

where $M$ denotes the bound on $\mathcal{H}^{N-1}\left(\left\{d_{K(t)}=\varepsilon\right\}\right)$ given by Proposition 2.13 Letting $n$ tend to $\infty$, we deduce that

$$
\int_{K^{\varepsilon}\left(t_{0}\right)} \phi(x) \mathrm{d} x \leqslant 0,
$$


which in view of the choice of $\phi$ implies that $x_{0} \notin K^{\varepsilon}\left(t_{0}\right)$, i.e. $d_{K\left(t_{0}\right)}\left(x_{0}\right) \geqslant \varepsilon=d_{K\left(t_{0}\right)}\left(x_{0}\right)+\delta$. This is absurd and proves the claim.

Step 2. A straightforward consequence of (3.13) is that, under assumption 3, we also have, for almost all $\varepsilon \in\left(0, \varepsilon_{0}\right), \sigma \in(0, \varepsilon)$, and for all $\phi \in C_{c}^{1}\left(\mathbb{R}^{N} \times(0, T), \mathbb{R}_{+}\right)$,

$$
\int_{0}^{T} \int_{K^{\sigma}(t)} \phi_{t}(x, t) \mathrm{d} x \mathrm{~d} t+\int_{0}^{T} \int_{\left\{d_{K(t)}=\sigma\right\}} c^{\varepsilon}(x, t) \phi(x, t) \mathrm{d} \mathcal{H}^{N-1}(x) \mathrm{d} t \geqslant 0,
$$

since $\phi \geqslant 0$ and $c^{\sigma} \leqslant c^{\varepsilon}$. Let us integrate inequality (4.2) for $\sigma$ between 0 and some fixed $\varepsilon \in\left(0, \varepsilon_{0}\right)$ and switch the order of integration between $t$ and $\sigma$ :

$\int_{0}^{T} \int_{0}^{\varepsilon} \int_{K^{\sigma}(t)} \phi_{t}(x, t) \mathrm{d} x \mathrm{~d} \sigma \mathrm{d} t+\int_{0}^{T} \int_{0}^{\varepsilon} \int_{\left\{d_{K(t)}=\sigma\right\}} c^{\varepsilon}(x, t) \phi(x, t) \mathrm{d} \mathcal{H}^{N-1}(x) \mathrm{d} \sigma \mathrm{d} t \geqslant 0$.

Now switching the order between $x$ and $\sigma$, we get

$$
\int_{0}^{\varepsilon} \int_{K^{\sigma}(t)} \phi_{t}(x, t) \mathrm{d} x \mathrm{~d} \sigma=\int_{K^{\varepsilon}(t)}\left(\varepsilon-d_{K(t)}(x)\right) \phi_{t}(x, t) \mathrm{d} x .
$$

Moreover, since $\left|D d_{K(t)}\right|=1$ a.e. on $\bigcup_{0<\sigma<\varepsilon}\left\{d_{K(t)}=\sigma\right\}=K^{\varepsilon}(t) \backslash K(t)$, we have, thanks to the co-area formula,

$$
\int_{0}^{\varepsilon} \int_{\left\{d_{K(t)}=\sigma\right\}} c^{\varepsilon}(x, t) \phi(x, t) \mathrm{d} \mathcal{H}^{N-1}(x) \mathrm{d} \sigma=\int_{K^{\varepsilon}(t) \backslash K(t)} c^{\varepsilon}(x, t) \phi(x, t) \mathrm{d} x,
$$

and we deduce from (4.3) that

$$
\int_{0}^{T} \int_{K^{\varepsilon}(t)}\left(\varepsilon-d_{K(t)}(x)\right) \phi_{t}(x, t) \mathrm{d} x \mathrm{~d} t+\int_{0}^{T} \int_{K^{\varepsilon}(t) \backslash K(t)} c^{\varepsilon}(x, t) \phi(x, t) \mathrm{d} x \mathrm{~d} t \geqslant 0 .
$$

Separating the test function into $(x, t) \mapsto \theta_{n}(t) \phi(x)$, with $\theta_{n}$ and $\phi$ of class $C^{1}$, and letting $\theta_{n} \rightarrow$ $\theta=\mathbf{1}_{\left[t_{1}, t_{2}\right]}$ with $0<t_{1}<t_{2}<T$, we deduce from (4.5) that

$$
\begin{aligned}
& \int_{K^{\varepsilon}\left(t_{2}\right)}\left(\varepsilon-d_{K\left(t_{2}\right)}(x)\right) \phi(x) \mathrm{d} x \\
& \quad \leqslant \int_{K^{\varepsilon}\left(t_{1}\right)}\left(\varepsilon-d_{K\left(t_{1}\right)}(x)\right) \phi(x) \mathrm{d} x+\int_{t_{1}}^{t_{2}} \int_{K^{\varepsilon}(t) \backslash K(t)} c^{\varepsilon}(x, t) \phi(x) \mathrm{d} x \mathrm{~d} t .
\end{aligned}
$$

This holds for almost all $t_{1}$ and $t_{2}$, and therefore for all $0<t_{1} \leqslant t_{2}<T$ thanks to Step 1 and the fact that all sets of the form $\left\{d_{K\left(t_{0}\right)}=\varepsilon\right\}$ have zero $\mathcal{L}^{N}$ measure. Seeing (4.6), we could be tempted to try to prove that $w:(x, t) \mapsto-d_{K(t)}(x)$ is a subsolution of $w_{t}=c^{\varepsilon}(x, t)|D w|$ on

$$
A_{\varepsilon}=\left\{(x, t) \in \mathbb{R}^{N} \times(0, T) ; 0<d_{K(t)}(x)<\varepsilon\right\}
$$

so that if $(\bar{x}, \bar{t}) \in A_{\varepsilon}$, then $\bar{x} \in K^{\varepsilon}(\bar{t}) \backslash K(\bar{t})$. Unfortunately, this last assertion does not make sense since $A_{\varepsilon}$ is not open. Indeed, $K(t)$ may "shrink" suddenly as $t$ increases. However, Step 1 implies that $A_{\varepsilon}$ is left open. Indeed, if $(x, t) \in A_{\varepsilon}$, then $s \mapsto d_{K(s)}(x)$ is left continuous at $t$ with $0<d_{K(t)}(x)<\varepsilon$. 
Let us now go back to 4.6, and show that $w:(x, t) \mapsto-d_{K(t)}(x)$ is a viscosity left subsolution of $w_{t}=c^{\varepsilon}(x, t)|D w|$ in $A_{\varepsilon}$. Take $(\bar{x}, \bar{t}) \in A_{\varepsilon}$ and $\psi$ of class $C^{1}$ on $\mathbb{R}^{N} \times(0, T)$ such that $w-\psi$ has a maximum equal to 0 on a left neighborhood of $(\bar{x}, \bar{t})$. Then for certain $r>0$ and $h>0$, and for all $(x, t) \in B(\bar{x}, r) \times[\bar{t}-h, \bar{t}] \subset A_{\varepsilon}$,

$$
-d_{K(t)}(x) \leqslant \psi(x, t),
$$

the inequality being an equality at $(\bar{x}, \bar{t})$. Taking $t_{1}=\bar{t}-h, t_{2}=\bar{t}$ in (4.6), and using the freedom in the choice of $\phi$ to let $\phi$ converge to the Dirac mass at $\bar{x}$ yields

$$
\varepsilon-d_{K(\bar{t})}(\bar{x}) \leqslant \varepsilon-d_{K(\bar{t}-h)}(\bar{x})+\int_{\bar{t}-h}^{\bar{t}} c^{\varepsilon}(\bar{x}, t) \mathrm{d} t .
$$

Then we can use (4.7) to obtain

$$
\psi(\bar{x}, \bar{t}) \leqslant \psi(\bar{x}, \bar{t}-h)+\int_{\bar{t}-h}^{\bar{t}} c^{\varepsilon}(\bar{x}, t) \mathrm{d} t .
$$

Dividing by $h>0$ and letting $h \rightarrow 0^{+}$gives $-\psi_{t}(\bar{x}, \bar{t})+c^{\varepsilon}(\bar{x}, \bar{t}) \geqslant 0$, which means that $w$ is a left subsolution of $w_{t}=c^{\varepsilon}(x, t)|D w|$ in $A_{\varepsilon}$, since $|D \psi(\bar{x}, \bar{t})|=1$. Indeed, $-d_{K(\bar{t})}$ is locally semiconvex around $\bar{x}$ thanks to Lemma 2.5 , and smaller than $\psi(\cdot, \bar{t})$, which is smooth, with equality at $\bar{x}$. Therefore $-d_{K(\bar{t})}$ is differentiable at $\bar{x}$ with $-D d_{K(\bar{t})}(\bar{x})=D \psi(\bar{x}, \bar{t})$, but $\left|D d_{K(\bar{t})}(\bar{x})\right|=1$ whenever this gradient exists.

As a consequence of the stability theorem, $\mathbf{1}_{\{w \geqslant-\varepsilon / 2\}}$ is a left subsolution of $w_{t}=c^{\varepsilon}(x, t)|D w|$ in $A_{\varepsilon}$, and therefore also in $\mathbb{R}^{N} \times(0, T)$. Indeed, if $x \notin K^{\varepsilon}(t)$, then $w(x, t)<-\frac{3}{4} \varepsilon$, which remains true in a neighborhood of $(x, t)$ since $w$ is usc. Thus $\mathbf{1}_{\{w \geqslant-\varepsilon / 2\}}(y, s)=0$ in a neighborhood of $(x, t)$. Moreover if $x \in K(t)$, then $w(x, t)>-\frac{1}{4} \varepsilon$, which this time does not necessarily hold on a neighborhood, but remains true on a left neighborhood of $(x, t)$ since $w$ is left continuous. Therefore $\mathbf{1}_{\{w \geqslant-\varepsilon / 2\}}(y, s)=1$ on a left neighborhood of $(x, t)$. In both cases, if $\mathbf{1}_{\{w \geqslant-\varepsilon / 2\}}-\psi$ has a maximum on a left neighborhood of $(x, t)$ with $\psi$ of class $C^{1}$, then $\psi_{t}(x, t) \leqslant 0=D \psi(x, t)$, so the equation is satisfied in the viscosity sense.

We have just proved that $\mathbf{1}_{\{w \geqslant-\varepsilon / 2\}}$ is a left subsolution of $w_{t}=c^{\varepsilon}(x, t)|D w|$ in $\mathbb{R}^{N} \times(0, T)$. But $\mathbb{R}^{N} \times(0, T)$ is open in the usual sense, so $\mathbf{1}_{\{w \geqslant-\varepsilon / 2\}}$ is actually a viscosity subsolution of $w_{t}=c^{\varepsilon}(x, t)|D w|$ in $\mathbb{R}^{N} \times(0, T)$ without restriction on the neighborhoods, since a local maximum is in particular a maximum on some left neighborhood.

Now since $w(x, t) \geqslant-\varepsilon / 2 \Leftrightarrow d_{K(t)}(x) \leqslant \varepsilon / 2$, the stability theorem shows, as $\varepsilon$ tends to 0 , that $u:(x, t) \mapsto \mathbf{1}_{K(t)}(x)$ is a subsolution of $u_{t}=c(x, t)|D u|$ on $\mathbb{R}^{N} \times(0, T)$, which is the desired conclusion. Indeed, $c^{\varepsilon}$ converges uniformly to $c$ since $c$ is Lipschitz continuous with respect to $x$, and if $\varepsilon_{n} \rightarrow 0^{+}$is fixed, then

$$
\mathbf{1}_{K(t)}(x)=\limsup \left\{\mathbf{1}_{\left\{d_{K\left(t_{n}\right)} \leqslant \varepsilon_{n} / 2\right\}}\left(x_{n}\right) ; t_{n} \rightarrow t, x_{n} \rightarrow x, \varepsilon_{n} \rightarrow 0\right\} .
$$

In order to see that, let us fix a sequence $\left(x_{n}, t_{n}\right)$ converging to $(x, t)$. We notice that if $x \in K(t)$, then $\mathbf{1}_{K(t)}(x)=1 \geqslant \sup \mathbf{1}_{\left\{d_{K\left(t_{n}\right)} \leqslant \varepsilon_{n} / 2\right\}}\left(x_{n}\right)$. Moreover if $x \notin K(t)$, then $(x, t)$ does not belong to the graph of $K$ which is closed, and thus is located at a positive distance from this graph, which implies that for $n$ large enough, $\mathbf{1}_{K(t)}(x)=0=\mathbf{1}_{\left\{d_{K\left(t_{n}\right)} \leqslant \varepsilon_{n} / 2\right\}}\left(x_{n}\right)$. Therefore

$$
\mathbf{1}_{K(t)}(x) \geqslant \lim \sup \left\{\mathbf{1}_{\left\{d_{K\left(t_{n}\right)} \leqslant \varepsilon_{n} / 2\right\}}\left(x_{n}\right) ; t_{n} \rightarrow t, x_{n} \rightarrow x, \varepsilon_{n} \rightarrow 0\right\} .
$$


To conclude it suffices to construct a sequence $\left(x_{n}, t_{n}\right)$ converging to $(x, t)$ such that $\mathbf{1}_{\left\{d_{K\left(t_{n}\right)} \leqslant \varepsilon_{n} / 2\right\}}\left(x_{n}\right) \rightarrow \mathbf{1}_{K(t)}(x)$. Take $x_{n}=x$ and $t_{n}=t$. If $x \notin K(t)$, we have just seen that $\mathbf{1}_{K(t)}(x)=0=\mathbf{1}_{\left\{d_{K(t)} \leqslant \varepsilon_{n} / 2\right\}}(x)$ for $n$ large enough. If $x \in K(t)$, then $x \in K^{\varepsilon_{n} / 2}(t)$ so that $\mathbf{1}_{\left\{d_{K(t)} \leqslant \varepsilon_{n} / 2\right\}}(x)=1=\mathbf{1}_{K(t)}(x)$.

\section{Regularity of the front}

In this section we use the integral formulation to derive estimates related to the regularity of $K(t)$, and more precisely its perimeter, by studying the limit of (3.2) as $\varepsilon$ tends to 0 . To this end we make the following assumptions in addition of those of Theorem 2.2 .

(A1) $c$ is of class $C^{1}, D c$ is locally Lipschitz continuous with respect to the space variable.

(A2) $D c(x, t) \neq 0$ if $c(x, t)=0$.

THEOREM 5.1 Let $c: \mathbb{R}^{N} \times[0, T] \rightarrow \mathbb{R}$ satisfy the assumptions of Theorem 2.2 and (A1), (A2). Let $K:[0, T] \rightarrow \mathcal{P}\left(\mathbb{R}^{N}\right) \backslash\{\emptyset\}$ be such that:

1. $K(0)$ is compact, $K(t) \rightarrow K(0)$ as $t \rightarrow 0$ and $K(t) \rightarrow K(T)$ as $t \rightarrow T$ in the Hausdorff distance.

2. The graph $\bigcup_{t \in[0, T]}\{t\} \times K(t)$ of $K$ is closed in $[0, T] \times \mathbb{R}^{N}$.

3. $u:(x, t) \mapsto \mathbf{1}_{K(t)}(x)$ is a subsolution of the eikonal equation 3.1].

Then the following hold:

(i) For a.a. $t \in[0, T], c(\cdot, t) \mathbf{1}_{K(t)}$ has bounded variation in $\{c(\cdot, t)<0\}$.

(ii) For a.a. $t \in[0, T], K(t)$ has locally finite perimeter in $\{c(\cdot, t)<0\}$.

(iii) Denoting by $(\cdot)_{-}$the negative part of a quantity $\left(x_{-}=\max (-x, 0)\right)$, we have

$$
\int_{0}^{T} \int_{\partial^{*} K(t)} c_{-}(x, t) \mathrm{d} \mathcal{H}^{N-1}(x) \mathrm{d} t<\infty,
$$

an upper bound for this integral being given by

$$
-\left[\int_{K(t)} \mathbf{1}_{\{c<0\}}(x, t) \mathrm{d} x\right]_{0}^{T}+\int_{0}^{T} \int_{K(t) \cap\{c(\cdot, t)=0\}} \frac{\left|c_{t}(x, t)\right|}{|D c(x, t)|} \mathbf{1}_{\left\{c_{t}<0\right\}}(x, t) \mathrm{d} \mathcal{H}^{N-1}(x) \mathrm{d} t .
$$

Proof of Theorem $5.1(i)$. we split this rather long proof into several lemmas. In what follows, $\bar{B}_{R}=$ $\bar{B}(0, R)$ will denote a large ball that contains $K^{\varepsilon}(t)$ for all $t \in[0, T]$ and $\varepsilon \leqslant 1$, and $\overline{K^{\varepsilon}}(t)$ stands for $\overline{K^{\varepsilon}(t)}$.

Lemma 5.2 For all $\theta>0$ small enough, for almost all $0<\varepsilon \leqslant \varepsilon_{0} \leqslant 1$,

$$
\begin{aligned}
\int_{0}^{T} & \int_{\overline{K^{\varepsilon}}(t) \cap\{c(\cdot, t)=-\theta\}} \frac{\left|c_{t}(x, t)\right|}{|D c(x, t)|} \mathbf{1}_{\left\{c_{t}<0\right\}}(x, t) \mathrm{d} \mathcal{H}^{N-1}(x) \mathrm{d} t \\
& +\int_{0}^{T} \int_{\left\{d_{K(t)=\varepsilon\}}\right.} \mathbf{1}_{\{c<-\theta\}}(x, t) c^{\varepsilon_{0}}(x, t) \mathrm{d} \mathcal{H}^{N-1}(x) \mathrm{d} t \geqslant\left[\int_{K^{\varepsilon}(t)} \mathbf{1}_{\{c<-\theta\}}(x, t) \mathrm{d} x\right]_{0}^{T} .
\end{aligned}
$$


Proof. Let us fix $\theta>0$ and $0<\varepsilon \leqslant \varepsilon_{0} \leqslant 1$ such that $(3.2)$ holds with this $\varepsilon$. For all $\eta \in(0, \theta)$, let $T_{\eta}$ be a smooth non-increasing function equal to 1 in $(-\infty,-\theta-\eta]$ and 0 in $[-\theta, \infty)$. Then (3.2) can be applied to $\phi_{\eta}=T_{\eta} \circ c$ with $t_{1}=0$ and $t_{2}=T$.

Now an approximation argument shows that (3.2) also holds for $T_{\eta}$ defined as follows:

1. $T_{\eta}$ is non-increasing and continuous,

2. $T_{\eta}(x)=1$ if $x \leqslant-\theta-\eta, \theta(x)=0$ if $x \geqslant-\theta$,

3. $T_{\eta}$ is affine on $[-\theta-\eta,-\theta]$,

and $\phi_{\eta}=T_{\eta} \circ c$. Since

$$
\left(\phi_{\eta}\right)_{t}=c_{t}\left(T_{\eta}^{\prime} \circ c\right)=-\frac{1}{\eta} c_{t} \mathbf{1}_{\{-\theta-\eta<c<-\theta\}},
$$

we get

$$
\begin{array}{r}
\int_{0}^{T} \int_{K^{\varepsilon}(t) \cap\{-\theta-\eta<c<-\theta\}}\left(-\frac{1}{\eta}\right) c_{t} \mathrm{~d} x \mathrm{~d} t+\int_{0}^{T} \int_{\left\{d_{K(t)}=\varepsilon\right\}} c^{\varepsilon}(x, t) \phi_{\eta}(x, t) \mathrm{d} \mathcal{H}^{N-1}(x) \mathrm{d} t \\
\geqslant\left[\int_{K^{\varepsilon}(t)} \phi_{\eta}(x, t) \mathrm{d} x\right]_{0}^{T} .
\end{array}
$$

We want to let $\eta$ tend to 0 in 5.2. Thanks to the co-area formula,

$$
\begin{aligned}
& \int_{0}^{T} \int_{K^{\varepsilon}(t) \cap\{-\theta-\eta<c<-\theta\}}\left(-\frac{1}{\eta}\right) c_{t}(x, t) \mathrm{d} x \mathrm{~d} t \\
& \quad \leqslant \int_{0}^{T} \int_{\overline{K^{\varepsilon}}(t) \cap\{-\theta-\eta<c<-\theta\}} \frac{1}{\eta}\left|c_{t}(x, t)\right| \mathbf{1}_{\left\{c_{t}<0\right\}}(x, t) \mathrm{d} x \mathrm{~d} t \\
&=\frac{1}{\eta} \int_{0}^{T} \int_{-\theta-\eta}^{-\theta} \int_{\overline{K^{\varepsilon}}(t) \cap\{c(\cdot, t)=\sigma\}} \frac{\left|c_{t}(x, t)\right|}{|D c(x, t)|} \mathbf{1}_{\left\{c_{t}<0\right\}}(x, t) \mathrm{d} \mathcal{H}^{N-1}(x) \mathrm{d} \sigma \mathrm{d} t .
\end{aligned}
$$

The co-area formula can be applied since $c$ is Lipschitz continuous with respect to the space variable and of class $C^{1}$, so that assumption (A2) implies that if $\theta$ is small enough and $\eta \in(0, \theta)$, then $D c$ is bounded away from 0 on $\{-\theta-\eta<c<-\theta\} \cap\left\{\bar{B}_{R} \times[0, T]\right\}$. As a standard consequence of assumptions (A1) and (A2), there exists $\sigma_{0}>0$ such that the map

$$
\sigma \mapsto \int_{0}^{T} \int_{\overline{K^{\varepsilon}}(t) \cap\{c(\cdot, t)=\sigma\}} \frac{\left|c_{t}(x, t)\right|}{|D c(x, t)|} \mathbf{1}_{\left\{c_{t}<0\right\}}(x, t) \mathrm{d} \mathcal{H}^{N-1}(x) \mathrm{d} t
$$

is usc on $\left(-\sigma_{0}, \sigma_{0}\right)$ for all $0 \leqslant \varepsilon \leqslant 1$ (with the convention that $K^{0}(t)=K(t)$ ). In particular for $\theta$ small enough, as $\eta$ tends to 0 , the last term of (5.3) satisfies

$$
\begin{aligned}
\limsup _{\eta \rightarrow 0} \frac{1}{\eta} \int_{0}^{T} \int_{-\theta-\eta}^{-\theta} \int_{\overline{K^{\varepsilon}}(t) \cap\{c(\cdot, t)=\sigma\}} \frac{\left|c_{t}(x, t)\right|}{|D c(x, t)|} \mathbf{1}_{\left\{c_{t}<0\right\}}(x, t) \mathrm{d} \mathcal{H}^{N-1}(x) \mathrm{d} \sigma \mathrm{d} t \\
\leqslant \int_{0}^{T} \int_{\overline{K^{\varepsilon}}(t) \cap\{c(\cdot, t)=-\theta\}} \frac{\left|c_{t}(x, t)\right|}{|D c(x, t)|} \mathbf{1}_{\left\{c_{t}<0\right\}}(x, t) \mathrm{d} \mathcal{H}^{N-1}(x) \mathrm{d} t .
\end{aligned}
$$


Moreover, for $\varepsilon \leqslant \varepsilon_{0}$,

$$
\begin{aligned}
\int_{0}^{T} \int_{\left\{d_{K(t)}=\varepsilon\right\}} c^{\varepsilon}(x, t) \phi_{\eta}(x, t) & \mathrm{d} \mathcal{H}^{N-1}(x) \mathrm{d} t \\
& \leqslant \int_{0}^{T} \int_{\left\{d_{K(t)=\varepsilon\}}\right.} c^{\varepsilon_{0}}(x, t) \phi_{\eta}(x, t) \mathrm{d} \mathcal{H}^{N-1}(x) \mathrm{d} t \\
& \underset{\eta \rightarrow 0}{\longrightarrow} \int_{0}^{T} \int_{\left\{d_{K(t)}=\varepsilon\right\}} \mathbf{1}_{\{c<-\theta\}}(x, t) c^{\varepsilon_{0}}(x, t) \mathrm{d} \mathcal{H}^{N-1}(x) \mathrm{d} t,
\end{aligned}
$$

and

$$
\left[\int_{K^{\varepsilon}(t)} \phi_{\eta}(x, t) \mathrm{d} x\right]_{0}^{T} \underset{\eta \rightarrow 0}{\longrightarrow}\left[\int_{K^{\varepsilon}(t)} \mathbf{1}_{\{c<-\theta\}}(x, t) \mathrm{d} x\right]_{0}^{T} .
$$

Combining (5.2) to (5.6) then gives 5.1).

Intermediate step. Let $\Omega_{t}=\left\{x \in \mathbb{R}^{N} ; c(x, t)<-\theta\right\}$ for $\theta$ chosen small enough so that (5.1) holds. Since $c$ is of class $C^{1}$, for $\varepsilon_{0} \leqslant 1$ small enough depending on $\theta, c^{\varepsilon_{0}}<0$ on $\bigcup_{t \in[0, T]}\left(\bar{B}_{R} \cap \Omega_{t}\right) \times\{t\}$, so $c^{\varepsilon_{0}}=-\left(c^{\varepsilon_{0}}\right)_{-}$on this set. Since in addition $c^{\varepsilon_{0}}$ is Lipschitz continuous with respect to the space variable because of its definition (with $\left\|D c^{\varepsilon_{0}}\right\|_{\infty} \leqslant\|D c\|_{\infty}$ ), by a regularization argument there exists $\bar{c}$ satisfying the following assumptions:

$$
\left\{\begin{array}{l}
\text { (i) } \bar{c} \text { is of class } C^{1} \text { on } \mathbb{R}^{N} \times[0, T] \\
\text { (ii) } \bar{c} \text { is Lipschitz continuous with respect to the space variable, } \\
\text { with }\|D \bar{c}\|_{\infty} \leqslant\left\|D c^{\varepsilon_{0}}\right\|_{\infty} \\
\text { (iii) } 0 \leqslant \bar{c} \leqslant\left(c^{\varepsilon_{0}}\right)_{-} \text {in } \bigcup_{t \in[0, T]}\left(\bar{B}_{R} \cap \Omega_{t}\right) \times\{t\}
\end{array}\right.
$$

Then 5.1 gives, for almost all $0<\varepsilon \leqslant \varepsilon_{0}$ and all $\bar{c}$ satisfying 5.7,

$$
\begin{aligned}
& \int_{0}^{T} \int_{\left\{d_{K(t)}=\varepsilon\right\}} \mathbf{1}_{\{c<-\theta\}}(x, t) \bar{c}(x, t) \mathrm{d} \mathcal{H}^{N-1}(x) \mathrm{d} t \\
& \leqslant \int_{0}^{T} \int_{\left\{d_{K(t)}=\varepsilon\right\}} \mathbf{1}_{\{c<-\theta\}}(x, t)\left(c^{\varepsilon_{0}}\right)_{-}(x, t) \mathrm{d} \mathcal{H}^{N-1}(x) \mathrm{d} t \\
& \leqslant-\left[\int_{K^{\varepsilon}(t)} \mathbf{1}_{\{c<-\theta\}}(x, t) \mathrm{d} x\right]_{0}^{T} \\
&+\int_{0}^{T} \int_{\left.\overline{K^{\varepsilon}}(t)\right) \cap\{c(\cdot, t)=-\theta\}} \frac{\left|c_{t}(x, t)\right|}{|D c(x, t)|} \mathbf{1}_{\left\{c_{t}<0\right\}}(x, t) \mathrm{d} \mathcal{H}^{N-1}(x) \mathrm{d} t .
\end{aligned}
$$

Let us fix $\bar{c}$ satisfying (5.7). Now we want, thanks to the control given by (5.8), to estimate the total variation of $\bar{c}(\cdot, t) \mathbf{1}_{K^{\varepsilon}(t)}$, and then let $\varepsilon$ tend 0 to get an estimate on $\bar{c}(\cdot, t) \mathbf{1}_{K(t)}$. To this end, we introduce $a_{\varepsilon}(x, t)=\bar{c}(x, t) \mathbf{1}_{K^{\varepsilon}(t)}(x)$. Proposition 3.2 of [3] on the product of a BV function and a regular function shows that for all $t \in[0, T]$ and all $\varepsilon>0, a_{\varepsilon}(\cdot, t) \in B V\left(\mathbb{R}^{N}\right)$, and if $\left[D a_{\varepsilon}(\cdot, t)\right]$ denotes the vector-valued variation measure of $a_{\varepsilon}(\cdot, t)$ as defined in Theorem 2.9, we have

$$
\left[D a_{\varepsilon}(\cdot, t)\right]=-\bar{c}(\cdot, t) v_{K^{\varepsilon}(t)} \mathcal{H}_{\left\lfloor\partial^{*} K^{\varepsilon}(t)\right.}^{N-1}+\mathbf{1}_{K^{\varepsilon}(t)} D \bar{c}(\cdot, t) \mathcal{L}^{N}
$$


Now let us introduce two notations:

1. For a fixed $t \in[0, T]$ and $\theta \geqslant 0, X_{t}^{\theta}$ denotes the set of vector-valued functions $\phi \in C^{1}\left(\bar{B}_{R}, \mathbb{R}^{N}\right)$ vanishing on $\bar{B}_{R} \cap \Omega_{t}^{c}$ with $\|\phi\|_{\infty} \leqslant 1$.

2. $X^{\theta}$ is the set of all functions $\phi$ such that $\phi(\cdot, t) \in X_{t}^{\theta}$ for all $t \in[0, T]$, with $\phi$ and $D \phi$ measurable on $\mathbb{R}^{N} \times[0, T]$, and $\|D \phi\|_{\infty}<\infty$.

LEMMA 5.3 For all $\theta>0$ small enough,

$$
\sup _{\phi \in X^{\theta}}\left\{\int_{0}^{T} \int_{K(t)} c_{-}(x, t) \mathbf{1}_{\{c<-\theta\}}(x, t) \operatorname{div}_{x} \phi(x, t) \mathrm{d} x \mathrm{~d} t\right\} \leqslant M(\theta),
$$

where $\operatorname{div}_{x}$ stands for the divergence with respect to the space variable, and where

$$
\begin{aligned}
M(\theta)= & \mathcal{L}^{N}(K(0))+T \mathcal{L}^{N}\left(\bar{B}_{R}\right)\|D c\|_{\infty} \\
& +\int_{0}^{T} \int_{K(t) \cap\{c(\cdot, t)=-\theta\}} \frac{\left|c_{t}(x, t)\right|}{|D c(x, t)|} \mathbf{1}_{\left\{c_{t}<0\right\}}(x, t) \mathrm{d} \mathcal{H}^{N-1}(x) \mathrm{d} t .
\end{aligned}
$$

Proof. Fix $\theta>0$ small enough and choose $\varepsilon_{0} \leqslant 1$ so that $(5.8)$ holds for almost all $\varepsilon \leqslant \varepsilon_{0}$ and all $\bar{c}$ satisfying (5.7). Let $\phi \in X^{\theta}$. Since $a_{\varepsilon}(\cdot, t)$ vanishes off $\bar{B}_{R}$ for $\varepsilon \leqslant \varepsilon_{0}$, we have thanks to (5.9), after extending $\phi(\cdot, t)$ to $\mathbb{R}^{N}$ in a $C^{1}$ way so as to obtain a function with compact support in the space variable,

$$
\begin{aligned}
\int_{0}^{T} \int_{\mathbb{R}^{N}} a_{\varepsilon}(x, t) & \operatorname{div}_{x} \phi(x, t) \mathrm{d} x \mathrm{~d} t \\
= & \int_{0}^{T} \int_{\partial^{*} K^{\varepsilon}(t)} \mathbf{1}_{\{c<-\theta\}}(x, t) \bar{c}(x, t)\left\langle\phi(x, t), v_{K^{\varepsilon}(t)}(x)\right\rangle \mathrm{d} \mathcal{H}^{N-1}(x) \mathrm{d} t \\
& -\int_{0}^{T} \int_{K^{\varepsilon}(t)} \mathbf{1}_{\{c<-\theta\}}(x, t)\langle D \bar{c}(x, t), \phi(x, t)\rangle \mathrm{d} x \mathrm{~d} t .
\end{aligned}
$$

The integration in time is justified since each of the expressions under the time integral signs is integrable on $(0, T)$ by Fubini's theorem. For $\phi \in X^{\theta}$,

$$
\begin{aligned}
\int_{0}^{T} \int_{\partial^{*} K^{\varepsilon}(t)} \mathbf{1}_{\{c<-\theta\}}(x, t) \bar{c}(x, t)\left\langle\phi(x, t), v_{K^{\varepsilon}(t)}(x)\right\rangle \mathrm{d} \mathcal{H}^{N-1}(x) \mathrm{d} t \\
\leqslant \int_{0}^{T} \int_{\left\{d_{K(t)}=\varepsilon\right\}} \mathbf{1}_{\{c<-\theta\}}(x, t) \bar{c}(x, t) \mathrm{d} \mathcal{H}^{N-1}(x) \mathrm{d} t
\end{aligned}
$$

since $\partial^{*} K^{\varepsilon}(t) \subset \partial K^{\varepsilon}(t)=\left\{d_{K(t)}=\varepsilon\right\}$, and $\mathbf{1}_{\{c<-\theta\}}(x, t) \bar{c}(x, t) \geqslant 0$ thanks to 5.7)(iii). But (5.8) gives an upper bound of this term for almost all $0<\varepsilon \leqslant \varepsilon_{0}$ :

$$
\begin{aligned}
& \int_{0}^{T} \int_{\left\{d_{K(t)}=\varepsilon\right\}} \mathbf{1}_{\{c<-\theta\}}(x, t) \bar{c}(x, t) \mathrm{d} \mathcal{H}^{N-1}(x) \mathrm{d} t \\
& \quad \leqslant \mathcal{L}^{N}\left(K^{\varepsilon_{0}}(0)\right)+\int_{0}^{T} \int_{\overline{K^{\varepsilon} 0}(t) \cap\{c(\cdot, t)=-\theta\}} \frac{\left|c_{t}(x, t)\right|}{|D c(x, t)|} \mathbf{1}_{\left\{c_{t}<0\right\}}(x, t) \mathrm{d} \mathcal{H}^{N-1}(x) \mathrm{d} t .
\end{aligned}
$$


In addition, for $\phi \in X^{\theta}$ and $\varepsilon \leqslant \varepsilon_{0}$,

$$
\begin{aligned}
& \left|\int_{0}^{T} \int_{K^{\varepsilon}(t)} \mathbf{1}_{\{c<-\theta\}}(x, t)\langle D \bar{c}(x, t), \phi(x, t)\rangle \mathrm{d} x \mathrm{~d} t\right| \\
& \leqslant T \mathcal{L}^{N}\left(\bar{B}_{R}\right)\|D \bar{c}\|_{\infty} \leqslant T \mathcal{L}^{N}\left(\bar{B}_{R}\right)\left\|D c^{\varepsilon_{0}}\right\|_{\infty} \leqslant T \mathcal{L}^{N}\left(\bar{B}_{R}\right)\|D c\|_{\infty}
\end{aligned}
$$

because of 5 5.7)(ii) and by definition of $c^{\varepsilon_{0}}$. Let us introduce a variation measure on $\{(x, t) \in$ $\left.\mathbb{R}^{N} \times[0, T] ; c(x, t)<-\theta\right\}$ with respect to the space variable by the following formula:

$$
\left\|D_{x} a_{\varepsilon}\right\|=\sup _{\phi \in X^{\theta}}\left\{\int_{0}^{T} \int_{\mathbb{R}^{N}} a_{\varepsilon}(x, t) \operatorname{div}_{x} \phi(x, t) \mathrm{d} x \mathrm{~d} t\right\} .
$$

We deduce from (5.11) to (5.14) that $\varepsilon \mapsto\left\|D_{x} a_{\varepsilon}\right\|$ is bounded on $\left(0, \varepsilon_{0}\right]$. Moreover $\left(a_{\varepsilon}\right)$ converges to $a:(x, t) \mapsto \bar{c}(x, t) \mathbf{1}_{K(t)}(x)$ in $L^{1}$ as $\varepsilon$ tends to 0 . But it is straightforward to see that $\left\|D_{x}(\cdot)\right\|$ is lsc for the topology of $L^{1}\left(\mathbb{R}^{N} \times(0, T)\right)$ (see [15, Theorem 1, p. 172]). This implies that $a$ has finite $\left\|D_{x}(\cdot)\right\|$ variation, with

$$
\begin{aligned}
\left\|D_{x} a\right\| \leqslant & \liminf _{\varepsilon \rightarrow 0}\left\|D_{x} a_{\varepsilon}\right\| \\
\leqslant & \mathcal{L}^{N}(K(0))+T \mathcal{L}^{N}\left(\bar{B}_{R}\right)\|D c\|_{\infty} \\
& +\int_{0}^{T} \int_{K(t) \cap\{c(\cdot, t)=-\theta\}} \frac{\left|c_{t}(x, t)\right|}{|D c(x, t)|} \mathbf{1}_{\left\{c_{t}<0\right\}}(x, t) \mathrm{d} \mathcal{H}^{N-1}(x) \mathrm{d} t=M(\theta) .
\end{aligned}
$$

The integral term in the last inequality comes when letting $\varepsilon_{0} \rightarrow 0$ in 5.13 by dominated convergence. Indeed, $\overline{K^{\varepsilon_{0}}}(t) \rightarrow K(t)$ everywhere and the domination comes from the fact that $D c$ is bounded away from 0 on the set $\{c=-\theta\} \cap\left\{\bar{B}_{R} \times[0, T]\right\}$. The inequality $\left\|D_{x} a\right\| \leqslant M(\theta)$ amounts to saying that for all $\bar{c}$ satisfying (5.7),

$$
\sup _{\phi \in X^{\theta}}\left\{\int_{0}^{T} \int_{K(t)} \bar{c}(x, t) \mathbf{1}_{\{c<-\theta\}}(x, t) \operatorname{div}_{x} \phi(x, t) \mathrm{d} x \mathrm{~d} t\right\} \leqslant M(\theta) .
$$

Now a convolution argument shows that we can make $\bar{c}$ converge uniformly to $\left(c^{\varepsilon_{0}}\right)_{-}$on $[0, T] \times$ $\bar{B}_{R}$ with $\bar{c}$ satisfying (5.7). Therefore going to the limit in the previous inequality by dominated convergence, and then finally letting $\varepsilon_{0} \rightarrow 0$ by the same argument, we get the result.

We are finally ready to let $\theta \rightarrow 0$. Set $X=X^{0}$ and for all $t \in[0, T], X_{t}=X_{t}^{0}$, where $X^{0}$ and $X_{t}^{0}$ are defined before Lemma 5.3 . In particular any $\phi \in X$ vanishes outside $\{c<0\}$.

LEMMA 5.4

$$
\sup _{\phi \in X}\left\{\int_{0}^{T} \int_{K(t)} c(x, t) \operatorname{div}_{x} \phi(x, t) \mathrm{d} x \mathrm{~d} t\right\} \leqslant M(0) .
$$

Proof. Let $\phi \in X$. Consider the truncation function $T^{\theta}$, a continuous and piecewise affine function, such that $T^{\theta}(s)=1$ for $s \leqslant-2 \theta$, and $T^{\theta}(s)=0$ for $s \geqslant-\theta$. We regularize $T^{\theta}$ to get a sequence $\left(T_{n}^{\theta}\right)$ of functions converging uniformly to $T^{\theta}$, whose derivatives converge pointwise to $\left(T^{\theta}\right)^{\prime}$, and such that there exists a constant $C>0$ satisfying $\left\|\left(T_{n}^{\theta}\right)^{\prime}\right\|_{\infty} \leqslant C / \theta$ for all $n$. Then (5.10) can be applied to $\left(T_{n}^{\theta} \circ c\right) \phi$. But

$$
\operatorname{div}_{x}\left(T_{n}^{\theta} \circ c\right) \phi=\left(\left(T_{n}^{\theta}\right)^{\prime} \circ c\right)\langle\phi, D c\rangle+\left(T_{n}^{\theta} \circ c\right) \operatorname{div}_{x} \phi .
$$


As $n$ goes to infinity, we deduce from the dominated convergence theorem that

$$
\begin{aligned}
& \int_{0}^{T} \int_{K(t)} c_{-}(x, t)\left(-\frac{1}{\theta}\right) \mathbf{1}_{\{-2 \theta<c<-\theta\}}(x, t)\langle\phi(x, t), D c(x, t)\rangle \mathrm{d} x \mathrm{~d} t \\
&+\int_{0}^{T} \int_{K(t)} c_{-}(x, t)\left(T^{\theta} \circ c\right) \operatorname{div}_{x} \phi(x, t) \mathrm{d} x \mathrm{~d} t \leqslant M(\theta) .
\end{aligned}
$$

As $\theta$ tends to 0 , the first term converges to 0 : the domination comes from the fact that $\left|c_{-}(x, t)\left(-\frac{1}{\theta}\right) \mathbf{1}_{\{-2 \theta<c<-\theta\}}(x, t)\right| \leqslant \frac{1}{\theta}(2 \theta)=2$ and the domain of integration is bounded. The second term converges to

$$
\int_{0}^{T} \int_{K(t)} c_{-}(x, t) \operatorname{div}_{x} \phi(x, t) \mathrm{d} x \mathrm{~d} t .
$$

Moreover, recall that $\theta \mapsto M(\theta)$ is usc on $\left(-\sigma_{0}, \sigma_{0}\right)$ for $\sigma_{0}$ small enough, which implies that $\lim \sup _{\theta \rightarrow 0} M(\theta) \leqslant M(0)$. Given that $c_{-}=-c$ on $\{c<0\}$, we get the desired result in the limit as $\theta \rightarrow 0$.

The following lemma is a consequence of (5.15) and will be proved at the end of this section for the sake of readability:

Lemma 5.5 For almost all $t \in[0, T]$,

$$
\sup _{\phi \in X_{t}}\left\{\int_{K(t)} c(x, t) \operatorname{div} \phi(x) \mathrm{d} x\right\}<\infty .
$$

We deduce from this lemma and the definition of $X_{t}$ that for almost all $t \in[0, T], c(\cdot, t) \mathbf{1}_{K(t)}$ has bounded variation in $\{c(\cdot, t)<0\}$, and assertion (i) of Theorem 5.1 is proved.

Proof of Theorem 5.1 ii . Fix a $t$ such that $c(\cdot, t) \mathbf{1}_{K(t)}$ has bounded variation in $\{c(\cdot, t)<0\}$. For all $\eta>0,1 / c(\cdot, t)$ is of class $C^{1}$ on $\left\{x \in \mathbb{R}^{N} ; c(x, t)<-\eta\right\}$ and Lipschitz continuous. It follows from Proposition 3.2 of [3] that $\mathbf{1}_{K(t)}$ has bounded variation, whence $K(t)$ has finite perimeter, in $\left\{x \in \mathbb{R}^{N} ; c(x, t)<-\eta\right\}$ for all $\eta>0$. This proves assertion (ii) of Theorem 5.1 $K(t)$ has locally finite perimeter in $\{c(\cdot, t)<0\}$ for almost all $t \in[0, T]$.

Proof of Theorem 5.1 (iii).

Step 1. As in the intermediate step before Lemma 5.3. let us fix $\theta>0$ small enough so that 5.1. holds, and take $\varepsilon_{0} \leqslant 1$ small enough depending on $\theta$ and $\bar{c}$ satisfying (5.7). Now (5.9) can be applied to $a:(x, t) \mapsto \bar{c}(x, t) \mathbf{1}_{K(t)}$ and shows that for almost all $t \in[0, T]$,

$$
[D a(\cdot, t)]=-\bar{c}(\cdot, t) v_{K(t)} \mathcal{H}_{\left\lfloor\partial^{*} K(t)\right.}^{N-1}+\mathbf{1}_{K(t)} D \bar{c}(\cdot, t) \mathcal{L}^{N} \quad \text { in }\{c(\cdot, t)<0\},
$$

which amounts to saying that for almost all $t \in[0, T]$, for all $\phi$ of class $C^{1}$ on $\mathbb{R}^{N}$ with compact support in $\{c(\cdot, t)<0\}$,

$$
\begin{aligned}
& \int_{K(t)} \bar{c}(x, t) \operatorname{div} \phi(x) \mathrm{d} x \\
&=\int_{\partial^{*} K(t)} \bar{c}(x, t)\left\langle\phi(x), \nu_{K(t)}(x)\right\rangle \mathrm{d} \mathcal{H}^{N-1}(x)-\int_{K(t)}\langle D \bar{c}(x, t), \phi(x)\rangle \mathrm{d} x .
\end{aligned}
$$


Now let $\phi$ be of class $C^{1}$ on $\bar{B}_{R}$ and vanish off $\{c(\cdot, t)<-\theta\}$ for some $\theta>0$. We can assume without changing any of the integrals in $(5.16)$ that $\phi$ has compact support in $\{c(\cdot, t)<0\}$, so that (5.16) holds for $\phi$.

Step 2. Fix $\phi \in X^{\theta}$. Thanks to 5.16 we have

$$
\begin{aligned}
& \int_{0}^{T} \int_{K(t)} \mathbf{1}_{\{c<-\theta\}}(x, t) \bar{c}(x, t) \operatorname{div}_{x} \phi(x, t) \mathrm{d} x \mathrm{~d} t \\
&=\int_{0}^{T} \int_{\partial^{*} K(t)} \mathbf{1}_{\{c<-\theta\}}(x, t) \bar{c}(x, t)\left\langle\phi(x, t), v_{K(t)}(x)\right\rangle \mathrm{d} \mathcal{H}^{N-1}(x) \mathrm{d} t \\
&-\int_{0}^{T} \int_{K(t)} \mathbf{1}_{\{c<-\theta\}}(x, t)\langle D \bar{c}(x, t), \phi(x, t)\rangle \mathrm{d} x \mathrm{~d} t .
\end{aligned}
$$

Let us go back to 5.11): by dominated convergence, the first and third terms of this equality respectively converge as $\varepsilon \rightarrow 0$ to the first and third terms of (5.17), which shows that

$$
\begin{aligned}
\int_{0}^{T} \int_{\partial^{*} K^{\varepsilon}(t)} \mathbf{1}_{\{c<-\theta\}}(x, t) \bar{c}(x, t)\left\langle\phi(x, t), v_{K^{\varepsilon}(t)}(x)\right\rangle \mathrm{d} \mathcal{H}^{N-1}(x) \mathrm{d} t \\
\underset{\varepsilon \rightarrow 0}{\longrightarrow} \int_{0}^{T} \int_{\partial^{*} K(t)} \mathbf{1}_{\{c<-\theta\}}(x, t) \bar{c}(x, t)\left\langle\phi(x, t), v_{K(t)}(x)\right\rangle \mathrm{d} \mathcal{H}^{N-1}(x) \mathrm{d} t .
\end{aligned}
$$

Therefore, letting $\varepsilon \rightarrow 0, \bar{c} \rightarrow\left(c^{\varepsilon_{0}}\right)_{-}$and $\varepsilon_{0} \rightarrow 0$ in estimate (5.8) now shows, since $\partial^{*} K^{\varepsilon}(t) \subset$ $\partial K^{\varepsilon}(t)=\left\{d_{K(t)}=\varepsilon\right\}$, that

$$
\begin{aligned}
& \int_{0}^{T} \int_{\partial^{*} K(t)} \mathbf{1}_{\{c<-\theta\}}(x, t) \bar{c}(x, t)\left\langle\phi(x, t), v_{K(t)}(x)\right\rangle \mathrm{d} \mathcal{H}^{N-1}(x) \mathrm{d} t \\
& \leqslant-\left[\int_{K(t)} \mathbf{1}_{\{c<-\theta\}}(x, t) \mathrm{d} x\right]_{0}^{T} \\
&+\int_{0}^{T} \int_{K(t) \cap\{c(, t)=-\theta\}} \frac{\left|c_{t}(x, t)\right|}{|D c(x, t)|} \mathbf{1}_{\left\{c_{t}<0\right\}}(x, t) \mathrm{d} \mathcal{H}^{N-1}(x) \mathrm{d} t .
\end{aligned}
$$

We will need two additional notations:

1. For a fixed $t \in[0, T]$ and $\theta \geqslant 0, \hat{X}_{t}^{\theta}$ denotes the set of vector-valued functions $\phi \in C^{0}\left(\bar{B}_{R}, \mathbb{R}^{N}\right)$ vanishing on $\bar{B}_{R} \cap \Omega_{t}^{c}$ with $\|\phi\|_{\infty} \leqslant 1$.

2. $\hat{X}^{\theta}$ is the set of all functions $\phi$ such that $\phi(\cdot, t) \in \hat{X}_{t}^{\theta}$ for all $t \in[0, T]$, with $\phi$ measurable on $\mathbb{R}^{N} \times[0, T]$.

An approximation argument shows that $(5.18)$ holds for $\phi \in \hat{X}^{\theta}$.

Step 3. We give a lemma in the spirit of Lemma 5.5. whose proof will also be given at the end of this section:

LEMMA 5.6 For all $\theta>0$ :

(i) For almost all $t \in[0, T]$,

$$
\sup _{\phi \in \hat{X}_{t}^{\theta}}\left\{\int_{\partial^{*} K(t)} c_{-}(x, t)\left\langle\phi(x), v_{K(t)}(x)\right\rangle \mathrm{d} \mathcal{H}^{N-1}(x)\right\}<\infty .
$$


(ii) Moreover,

$$
\begin{aligned}
\sup _{\phi \in \hat{X}^{\theta}}\left\{\int_{0}^{T} \int_{\partial^{*} K(t)} c_{-}(x, t)\left\langle\phi(x, t), v_{K(t)}(x)\right\rangle \mathrm{d} \mathcal{H}^{N-1}(x) \mathrm{d} t\right\} \\
=\int_{0}^{T} \sup _{\phi \in \hat{X}_{t}^{\theta}}\left\{\int_{\partial^{*} K(t)} c_{-}(x, t)\left\langle\phi(x), v_{K(t)}(x)\right\rangle \mathrm{d} \mathcal{H}^{N-1}(x)\right\} \mathrm{d} t .
\end{aligned}
$$

We keep in mind that 5.18 gives an upper bound for the left-hand side of this equality. Now we show that for all $\theta>0$ and $t \in[0, T]$ such that $(5.19)$ holds and $K(t)$ has finite perimeter in $\left\{x \in \mathbb{R}^{N} ; c(x, t)<-\theta\right\}$ (which is true for almost all $t$ thanks to the proof of Theorem 5.1 (ii)), the supremum actually equals

$$
\int_{\partial^{*} K(t)} c_{-}(x, t) \mathbf{1}_{\{c<-\theta\}}(x, t) d \mathcal{H}^{N-1}(x) .
$$

Let such $\theta>0$ and $t \in[0, T]$ be fixed. Let $C \subset\{c(\cdot, t)<-\theta\}$ be compact. Since $K(t)$ has finite perimeter in $\left\{x \in \mathbb{R}^{N} ; c(x, t)<-\theta\right\}, C \cap \partial^{*} K(t)$ is a set of finite $\mathcal{H}^{N-1}$ measure. Therefore the integral

$$
\int_{C \cap \partial^{*} K(t)} c_{-}(x, t) \mathrm{d} \mathcal{H}^{N-1}
$$

is finite and according to Lusin's theorem (see [15] for instance), we know that for all $n \geqslant 1$, there exists a compact set $C_{n} \subset C \cap \partial^{*} K(t)$ such that $\nu_{K(t)}$ restricted to $C_{n}$ is continuous and

$$
\mathcal{H}^{N-1}\left(\left[C \cap \partial^{*} K(t)\right] \backslash C_{n}\right)<1 / n .
$$

We fix $n \geqslant 1$. For all $\eta>0$ small enough so that $C+B(0, \eta) \subset\{c(\cdot, t)<-\theta\}$, we consider $\phi_{\eta}$ such that $\phi_{\eta} \in C_{c}^{0}\left(C_{n}+B(0, \eta)\right),\left\|\phi_{\eta}\right\| \leqslant 1$ and $\phi_{\eta}$ coincides with $v_{K(t)}$ on $C_{n}$. The existence of $\phi_{\eta}$ is guaranteed by [15, Theorem 1, p. 13]. Then $\phi_{\eta} \in \hat{X}_{t}^{\theta}$ and

$$
\begin{aligned}
& \int_{\partial^{*} K(t)} c_{-}(x, t)\left\langle\phi_{\eta}(x), v_{K(t)}(x)\right\rangle \mathrm{d} \mathcal{H}^{N-1}(x) \\
& =\int_{C_{n}} c_{-}(x, t) \mathrm{d} \mathcal{H}^{N-1}(x)+\int_{\partial^{*} K(t)} \mathbf{1}_{\left[C_{n}+B(0, \eta)\right] \backslash C_{n}}(x) c_{-}(x, t)\left\langle\phi_{\eta}(x), v_{K(t)}(x)\right\rangle \mathrm{d} \mathcal{H}^{N-1}(x) .
\end{aligned}
$$

As $\eta$ goes to 0 ,

$$
\begin{array}{r}
\left|\int_{\partial^{*} K(t)} \mathbf{1}_{\left[C_{n}+B(0, \eta)\right] \backslash C_{n}}(x) c_{-}(x, t)\left\langle\phi_{\eta}(x), v_{K(t)}(x)\right\rangle \mathrm{d} \mathcal{H}^{N-1}(x)\right| \\
\leqslant\|c\|_{\infty} \int_{\partial^{*} K(t)} \mathbf{1}_{\left[C_{n}+B(0, \eta)\right] \backslash C_{n}}(x) d \mathcal{H}^{N-1}(x) \rightarrow 0
\end{array}
$$

thanks to the dominated convergence theorem. Therefore

$$
\begin{aligned}
\mid \int_{\partial^{*} K(t)} c_{-}(x, t)\left\langle\phi_{\eta}(x), v_{K(t)}(x)\right\rangle \mathrm{d} \mathcal{H}^{N-1}(x) & -\int_{C \cap \partial^{*} K(t)} c_{-}(x, t) \mathrm{d} \mathcal{H}^{N-1}(x) \mid \\
& \underset{\eta \rightarrow 0}{\longrightarrow} \int_{\left[C \cap \partial^{*} K(t)\right] \backslash C_{n}} c_{-}(x, t) \mathrm{d} \mathcal{H}^{N-1}(x) \leqslant\|c\|_{\infty} \frac{1}{n}
\end{aligned}
$$


thanks to [5.22]. We have constructed functions $\phi_{\eta} \in \hat{X}_{t}^{\theta}$ such that the integral

$$
\int_{\partial^{*} K(t)} c_{-}(x, t)\left\langle\phi_{\eta}(x), v_{K(t)}(x)\right\rangle \mathrm{d} \mathcal{H}^{N-1}(x)
$$

is arbitrarily close to the integral $\int_{C \cap \partial^{*} K(t)} c_{-}(x, t) \mathrm{d} \mathcal{H}^{N-1}(x)$, which in turn, by taking $C=C_{p}=$ $\bar{B}(0, p) \cap\left\{x ; d_{\{c(\cdot, t) \geqslant-\theta\}}(x) \geqslant 1 / p\right\}$, as $p \rightarrow \infty$ by monotone convergence can be made arbitrarily close to $\int_{\partial^{*} K(t)} c_{-}(x, t) \mathbf{1}_{\{c<-\theta\}}(x, t) \mathrm{d} \mathcal{H}^{N-1}(x)$. This proves [5.21].

Step 4. Combining the results of Steps 2 and 3, we have, for all $\theta>0$,

$$
\begin{aligned}
& \int_{0}^{T} \int_{\partial^{*} K(t)} c_{-}(x, t) \mathbf{1}_{\{c<-\theta\}}(x, t) \mathrm{d} \mathcal{H}^{N-1}(x) \mathrm{d} t \\
& \quad \leqslant-\left[\int_{K(t)} \mathbf{1}_{\{c<-\theta\}}(x, t) \mathrm{d} x\right]_{0}^{T}+\int_{0}^{T} \int_{K(t) \cap\{c(\cdot, t)=-\theta\}} \frac{\left|c_{t}(x, t)\right|}{|D c(x, t)|} \mathbf{1}_{\left\{c_{t}<0\right\}}(x, t) \mathrm{d} \mathcal{H}^{N-1}(x) \mathrm{d} t .
\end{aligned}
$$

If we let $\theta \rightarrow 0$ in this estimate, since the right-hand side is upper semicontinuous, the Beppo-Levi monotone convergence theorem shows that $\int_{0}^{T} \int_{\partial^{*} K(t)} c_{-}(x, t) \mathrm{d} \mathcal{H}^{N-1}(x) \mathrm{d} t$ is finite and

$$
\begin{aligned}
& \int_{0}^{T} \int_{\partial^{*} K(t)} c_{-}(x, t) \mathrm{d} \mathcal{H}^{N-1}(x) \mathrm{d} t \\
& \leqslant-\left[\int_{K(t)} \mathbf{1}_{\{c<0\}}(x, t) \mathrm{d} x\right]_{0}^{T}+\int_{0}^{T} \int_{K(t) \cap\{c(\cdot, t)=0\}} \frac{\left|c_{t}(x, t)\right|}{|D c(x, t)|} \mathbf{1}_{\left\{c_{t}<0\right\}}(x, t) \mathrm{d} \mathcal{H}^{N-1}(x) \mathrm{d} t,
\end{aligned}
$$

and this finally proves the third assertion of Theorem 5.1

Proof of Lemma 5.5. If the lemma were not true, there would exist $A \subset[0, T]$ with positive measure such that for all $t \in A, \sup _{\phi \in X_{t}}\left\{\int_{K(t)} c(x, t) \operatorname{div} \phi(x) \mathrm{d} x\right\}=\infty$. Let $\varepsilon>0$ be fixed. For all $M>0$, set

$$
E_{M}=\left\{t \in A ; \exists \phi \in X_{t} \text { with }\|D \phi\|_{\infty} \leqslant M, \int_{K(t)} c(x, t) \operatorname{div} \phi(x) \mathrm{d} x>1 / \varepsilon\right\},
$$

which is non-empty for $M$ large enough. We also point out that $A=\bigcup_{M>0} E_{M}$. Let us fix $M$ large enough so that $E_{M} \neq \emptyset$. We want to construct $\phi \in X$ such that $\|D \phi\|_{\infty} \leqslant M$ and for all $t \in E_{M}, \int_{K(t)} c(x, t) \operatorname{div} \phi(x, t) \mathrm{d} x>1 / \varepsilon$. In order to do so, we will need the notion of measurable selection. For more details than those given below, we refer to [4].

We recall that if $(\Omega, \mathcal{A})$ is a measurable space, and $Y$ is a complete separable metric space, a map $F: \Omega \rightarrow \mathcal{P}(Y)$ whose images are closed subsets of $Y$ is said to be measurable if for all open subsets $U \subset Y, F^{-1}(U)=\{\omega \in \Omega ; F(\omega) \cap U \neq \emptyset\} \in \mathcal{A}$. A measurable map $f: \Omega \rightarrow Y$ such that for all $\omega \in \Omega, f(\omega) \in F(\omega)$, is called a measurable selection of $F$.

If $Z$ is another complete separable metric space, we say that $g: \Omega \times Y \rightarrow Z$ is Carathéodory if for all $\omega \in \Omega, g(\omega, \cdot)$ is continuous, and for all $x \in Y, g(\cdot, x)$ is measurable. In order to construct our function $\phi$, we use the following measurable selection theorem:

THEOREM 5.7 ([4, Theorem 8.2.9]) Let $(\Omega, \mathcal{A})$ be a measurable space. Let $Y$ and $Z$ be two complete separable metric spaces, and $F: \Omega \rightarrow \mathcal{P}(Y)$ and $G: \Omega \rightarrow \mathcal{P}(Z)$ be two measurable 
maps with closed images. Let $g: \Omega \times Y \rightarrow Z$ be a Carathéodory map. Then the map $\omega \mapsto\{x \in$ $F(\omega) ; g(\omega, x) \in G(\omega)\}$ is measurable. Moreover if

$$
\forall \omega \in \Omega, \quad g(\omega, F(\omega)) \cap G(\omega) \neq \emptyset,
$$

then there exists a measurable selection $f$ of $F$ such that for all $\omega \in \Omega, g(\omega, f(\omega)) \in G(\omega)$.

We apply Theorem 5.7 with

$$
\begin{aligned}
& \Omega=E_{M}, \quad Z=\mathbb{R}, \\
& Y=\left\{\phi \in C^{1}\left(\bar{B}_{R}\right) ;\|\phi\|_{\infty} \leqslant 1 \text { and } \mid D \phi \|_{\infty} \leqslant M\right\} \quad \text { with the norm }\|\phi\|_{\infty}+\|D \phi\|_{\infty}, \\
& F(t)=\left\{\phi \in X_{t} ;\|D \phi\|_{\infty} \leqslant M\right\}, \quad G(t)=[1 / \varepsilon, \infty), \\
& g(t, \phi)=\int_{K(t)} c(x, t) \operatorname{div} \phi(x) \mathrm{d} x .
\end{aligned}
$$

We first notice that $Y$ and $Z$ are complete separable metric spaces, and that $F$ and $G$ have closed images. Moreover, $G$ is constant, thus measurable.

1. For all $t \in[0, T], F(t)=\left\{\phi \in Y\right.$; $\left.\sup _{\bar{B}_{R} \cap\{c(\cdot, t) \geqslant 0\}}|\phi(x)|=0\right\}$, and Theorem 5.7 applied to $F_{1}(t)=Y, G_{1}(t)=\{0\}, g_{1}(t, \phi)=\sup _{\bar{B}_{R} \cap\{c(\cdot, t) \geqslant 0\}}|\phi(x)|$ shows that $F$ is measurable on [0,T]. Indeed $g_{1}$ is Carathéodory: $g_{1}(\cdot, \phi)$ is clearly usc, whence measurable.

2. Next we see that $t \mapsto g(t, \phi)$ is measurable on $[0, T]$ for all $\phi \in C^{1}\left(\bar{B}_{R}\right)$, because of Fubini's theorem, since $(x, t) \mapsto \mathbf{1}_{K(t)}(x) c(x, t) \operatorname{div} \phi(x) \in L^{1}\left(\mathbb{R}^{N} \times[0, T]\right)$. Moreover $\phi \mapsto g(t, \phi)$ is continuous since the domain of integration is bounded and $c$ is bounded, which shows that $g$ is Carathéodory.

3. The supremum of measurable functions on a set that depends in a measurable way on $t$ is measurable (cf. [4, Theorem 8.2.11]), so Steps 1 and 2 show that

$$
h: t \mapsto \sup _{\phi \in F(t)}\left\{\int_{K(t)} c(x, t) \operatorname{div} \phi(x) \mathrm{d} x\right\}
$$

is measurable on [0,T]. But $E_{M}=h^{-1}((1 / \varepsilon, \infty)) \cap A$, hence $E_{M}$ is Lebesgue measurable. By restriction, the maps $F, G$ and $g(\cdot, \phi)(\forall \phi \in Y)$ are measurable on $E_{M}$.

The assumptions of the measurable selection theorem are satisfied, and after extending off $E_{M}$ by multiplying the measurable selection obtained by $\mathbf{1}_{E_{M}}$ which is measurable, we get the existence of $\phi \in X$ such that for all $t \in E_{M}$,

$$
\int_{K(t)} c(x, t) \operatorname{div}_{x} \phi(x, t) \mathrm{d} x \geqslant 1 / \varepsilon,
$$

and this integral vanishes on $[0, T] \backslash E_{M}$. Integrating this inequality between 0 and $T$, taking the supremum for $\phi \in X$ on the left-hand side, and letting $M$ go to infinity we conclude, since $A=$ $\bigcup_{M>0} E_{M}$, that

$$
\sup _{\phi \in X}\left\{\int_{0}^{T} \int_{K(t)} c(x, t) \operatorname{div}_{x} \phi(x, t) \mathrm{d} x \mathrm{~d} t\right\} \geqslant \frac{\mathcal{L}(A)}{\varepsilon} .
$$

Since this is true for all $\varepsilon>0$, and since $\mathcal{L}(A)>0$, letting $\varepsilon \rightarrow 0$ contradicts 5.15 and concludes the proof of Lemma 5.5 . 
Proof of Lemma $5.6(i)$. The proof is in every way similar to the previous one, with the following modifications:

1. We need not consider $E_{M}$ since there is no first order derivative condition. So $\Omega$ is the set of $t \in[0, T]$ such that $K(t)$ has finite perimeter in $\{c(\cdot, t)<0\}$, which is known to have full measure.

2. $C^{1}\left(\bar{B}_{R}\right)$ is replaced by $C^{0}\left(\bar{B}_{R}\right)$ with the $\|\cdot\|_{\infty}$ norm, and $X_{t}$ is replaced by $\hat{X}_{t}^{\theta}$.

3. $g$ is defined by $g(t, \phi)=\int_{\partial^{*} K(t)} c_{-}(x, t)\left\langle\phi(x, t), v_{K(t)}(x)\right\rangle \mathrm{d} \mathcal{H}^{N-1}(x)$, the main argument to verify that $g$ is Carathéodory being that $\mathcal{H}^{N-1}\left(\{c(\cdot, t)<-\theta\} \cap \partial^{*} K(t)\right)$ is finite for all $t \in \Omega$.

Proof of Lemma 5.6 (ii). We start by noticing that the " $\leqslant$ " inequality of $(5.20)$ is obvious. Now we know, thanks to (i), that there exists $A$ of zero measure such that for $t \in \Omega \backslash A$,

$$
\sup _{\phi \in \hat{X}_{t}^{\theta}}\left\{\int_{\partial^{*} K(t)} c_{-}(x, t)\left\langle\phi(x), v_{K(t)}(x)\right\rangle \mathrm{d} \mathcal{H}^{N-1}(x)\right\}<\infty .
$$

Let $\varepsilon>0$ be fixed. We apply Theorem 5.7 with

$$
\begin{aligned}
& Y=\left\{\phi \in C^{0}\left(\bar{B}_{R}\right) ;\|\phi\|_{\infty} \leqslant 1\right\} \quad \text { with the norm }\|\phi\|_{\infty}, \quad Z=\mathbb{R}, \\
& F(t)=\hat{X}_{t}^{\theta}, \\
& G(t)=\left[\sup _{\phi \in \hat{X}_{t}^{\theta}}\left\{\int_{\partial^{*} K(t)} c_{-}(x, t)\left\langle\phi(x), v_{K(t)}(x)\right\rangle \mathrm{d} \mathcal{H}^{N-1}(x)\right\}-\varepsilon, \infty\right), \\
& g(t, \phi)=\int_{\partial^{*} K(t)} c_{-}(x, t)\left\langle\phi(x), v_{K(t)}(x)\right\rangle \mathrm{d} \mathcal{H}^{N-1}(x) .
\end{aligned}
$$

All the maps considered are measurable thanks to the previous proof. Indeed, another use of Theorem 5.7 with $Y_{1}=Z_{1}=\mathbb{R}, F_{1}(t)=\mathbb{R}, G_{1}(t)=[0, \infty)$, and $g_{1}(y, t)=y-$ $\sup _{\phi \in \hat{X}_{t}^{\theta}}\left\{\int_{\partial^{*} K(t)} c_{-}(x, t)\left\langle\phi(x), v_{K(t)}(x)\right\rangle \mathrm{d} \mathcal{H}^{N-1}(x)\right\}+\varepsilon$ shows that $G$ is measurable, which was the only missing verification.

As in the proof of Lemma 5.5, we obtain the existence of $\phi \in \hat{X}^{\theta}$ such that for all $t \in \Omega \backslash A$,

$$
\int_{K(t)} c(x, t) \operatorname{div}_{x} \phi(x, t) \mathrm{d} x \geqslant \sup _{\phi \in \hat{X}_{t}^{\theta}}\left\{\int_{K(t)} c(x, t) \operatorname{div} \phi(x) \mathrm{d} x\right\}-\varepsilon .
$$

Integrating this inequality between 0 and $T$, and then letting $\varepsilon \rightarrow 0$ proves 5.20 .

\section{Corresponding results for supersolutions}

We state in this section the counterparts for supersolutions of the main results proved up to now, and give the modifications needed for the proofs.

\subsection{The integral formulation for supersolutions}

Let $c: \mathbb{R}^{N} \times[0, T] \rightarrow \mathbb{R}$ satisfy the assumptions of Theorem 2.2 and $\Omega:[0, T] \rightarrow \mathcal{P}\left(\mathbb{R}^{N}\right) \backslash\left\{\mathbb{R}^{N}\right\}$ be such that

1. $\Omega$ is uniformly bounded on [0,T], $\Omega(t) \rightarrow \Omega(0)$ as $t \rightarrow 0$ and $\Omega(t) \rightarrow \Omega(T)$ as $t \rightarrow T$ in the Hausdorff distance. 
2. The graph $\bigcup_{t \in[0, T]}\{t\} \times \Omega(t)$ of $\Omega$ is open in $[0, T] \times \mathbb{R}^{N}$.

3. $u:(x, t) \mapsto \mathbf{1}_{\Omega(t)}(x)$ is a viscosity supersolution of the eikonal equation

$$
u_{t}=c(x, t)|D u| \quad \text { in } \mathbb{R}^{N} \times(0, T) .
$$

Set for all $\varepsilon>0$,

$$
\Omega^{\varepsilon}(t)=\left\{x \in \mathbb{R}^{N} ; d_{\Omega^{c}(t)}(x)>\varepsilon\right\}, \quad c_{\varepsilon}(x, t)=\min _{|y-x| \leqslant \varepsilon} c(y, t) .
$$

Then we have:

THEOREM 6.1 For all $t_{1}$ and $t_{2}$ satisfying $0 \leqslant t_{1} \leqslant t_{2} \leqslant T$, for almost all $\varepsilon>0$, and for all $\phi \in C^{1}\left(\mathbb{R}^{N} \times[0, T], \mathbb{R}_{+}\right)$,

$$
\begin{aligned}
\int_{t_{1}}^{t_{2}} \int_{\Omega^{\varepsilon}(t)} \phi_{t}(x, t) \mathrm{d} x \mathrm{~d} t+\int_{t_{1}}^{t_{2}} \int_{\left\{d_{\Omega^{c}(t)}=\varepsilon\right\}} c_{\varepsilon}(x, t) \phi(x, t) \mathrm{d} \mathcal{H}^{N-1}(x) \mathrm{d} t & \\
& \leqslant\left[\int_{\Omega^{\varepsilon}(t)} \phi(x, t) \mathrm{d} x\right]_{t_{1}}^{t_{2}} .
\end{aligned}
$$

REMARK 6.2 The fact that $u$ is a supersolution of the equation does not guarantee, unlike the case of subsolutions, that the evolution is bounded. This is why assumption 1 has to be stronger for supersolutions.

The only changes in the proof are:

1. $w(x, t)=d_{\Omega^{c}(t)}(x)$.

2. We take $\theta: \mathbb{R} \rightarrow \mathbb{R}$ non-decreasing of class $C^{\infty}$ such that $\theta=0$ in $(-\infty, 0], \theta=1$ in $[\varepsilon, \infty)$.

3. The equivalent of Proposition 3.5 is proved by a regularization by inf-convolution instead of sup-convolution.

All arguments then follow in the same way.

\subsection{The converse implication for supersolutions}

The converse theorem for supersolutions naturally becomes:

THEOREM 6.3 Let $c: \mathbb{R}^{N} \times[0, T] \rightarrow \mathbb{R}$ satisfy the assumptions of Theorem 2.2 and $\Omega:[0, T] \rightarrow$ $\mathcal{P}\left(\mathbb{R}^{N}\right) \backslash\left\{\mathbb{R}^{N}\right\}$ be such that

1. $\Omega$ is uniformly bounded on $[0, T]$.

2. The graph $\bigcup_{t \in[0, T]}\{t\} \times \Omega(t)$ of $\Omega$ is open in $[0, T] \times \mathbb{R}^{N}$.

3. Inequality $(6.1)$ holds for all $0 \leqslant t_{1} \leqslant t_{2} \leqslant T$, for almost all $\varepsilon>0$ small enough and for all $\phi \in C^{1}\left(\mathbb{R}^{N} \times[0, T], \mathbb{R}_{+}\right)$.

Then $u:(x, t) \mapsto \mathbf{1}_{\Omega(t)}(x)$ is a viscosity supersolution of $u_{t}=c(x, t)|D u|$ in $\mathbb{R}^{N} \times(0, T)$.

In the proof of this result, the only change occurs when switching the integration order between $x$ and $\sigma$ (the equivalent of (4.4)), and we get

$$
\int_{0}^{\varepsilon} \int_{\Omega^{\sigma}(t)} \phi_{t}(x, t) \mathrm{d} x \mathrm{~d} \sigma=\int_{\Omega(t)} d_{\Omega^{c}(t)}(x) \phi_{t}(x, t) \mathrm{d} x,
$$

which is exactly what we need to comply with the inequalities for supersolutions. 


\subsection{Regularity of the front}

The analogue of Theorem 5.1 when we work on 6.1 is the following:

THEOREM 6.4 Let $c: \mathbb{R}^{N} \times[0, T] \rightarrow \mathbb{R}$ satisfy the assumptions of Theorem 2.2 and (A1), (A2). Let $\Omega:[0, T] \rightarrow \mathcal{P}\left(\mathbb{R}^{N}\right) \backslash\left\{\mathbb{R}^{N}\right\}$ be such that:

1. $\Omega$ is uniformly bounded on [0,T], $\Omega(t) \rightarrow \Omega(0)$ as $t \rightarrow 0$ and $\Omega(t) \rightarrow \Omega(T)$ as $t \rightarrow T$ in the Hausdorff distance.

2. The graph $\bigcup_{t \in[0, T]}\{t\} \times \Omega(t)$ of $\Omega$ is open in $[0, T] \times \mathbb{R}^{N}$.

3. $u:(x, t) \mapsto \mathbf{1}_{\Omega(t)}(x)$ is a supersolution of the eikonal equation (3.1).

Then the following hold:

(i) For a.a. $t \in[0, T], c(\cdot, t) \mathbf{1}_{\Omega(t)}$ has bounded variation in $\{c(\cdot, t)>0\}$.

(ii) For a.a. $t \in[0, T], \Omega(t)$ has locally finite perimeter in $\{c(\cdot, t)>0\}$.

(iii) Denoting by $(\cdot)_{+}$the positive part of a quantity $\left(x_{+}=\max (x, 0)\right)$, we have

$$
\int_{0}^{T} \int_{\partial^{*} \Omega(t)} c_{+}(x, t) \mathrm{d} \mathcal{H}^{N-1}(x) \mathrm{d} t<\infty,
$$

an upper bound for this integral being given by

$$
\left[\int_{\Omega(t)} \mathbf{1}_{\{c>0\}}(x, t) \mathrm{d} x\right]_{0}^{T}+\int_{0}^{T} \int_{\Omega(t) \cap\{c(\cdot, t)=0\}} \frac{\left|c_{t}(x, t)\right|}{|D c(x, t)|} \mathbf{1}_{\left\{c_{t}<0\right\}}(x, t) d \mathcal{H}^{N-1}(x) \mathrm{d} t .
$$

The only modification is basically to switch all $\{c<0\}$ to $\{c>0\}$ and use the modified truncation $T_{\eta}$, a continuous non-decreasing and piecewise affine function equal to 0 in $(-\infty, \theta]$ and 1 in $[\theta+\eta, \infty)$ so as to isolate the set $\{c>0\}$ instead of $\{c<0\}$.

\section{Global estimate}

We finally synthesize the results of Theorems 5.1 and 6.4 in order to get a global estimate.

THEOREM 7.1 Let $c: \mathbb{R}^{N} \times[0, T] \rightarrow \mathbb{R}$ satisfy the assumptions of Theorem 2.2 and (A1), (A2), and let $\Omega:[0, T] \rightarrow \mathcal{P}\left(\mathbb{R}^{N}\right)$ and $K:[0, T] \rightarrow \mathcal{P}\left(\mathbb{R}^{N}\right) \backslash\{\emptyset\}$ be such that:

1. $K(0)$ is compact and for all $t \in[0, T], \Omega(t) \subset K(t)$.

2. $K(t) \rightarrow K(0)$ and $\Omega(t) \rightarrow \Omega(0)$ in the Hausdorff distance as $t \rightarrow 0$, $K(t) \rightarrow K(T)$ and $\Omega(t) \rightarrow \Omega(T)$ in the Hausdorff distance as $t \rightarrow T$.

3. $\operatorname{Graph}(K)$ is closed in $[0, T] \times \mathbb{R}^{N}$ and $\underline{u}:(x, t) \mapsto \mathbf{1}_{K(t)}(x)$ is a subsolution of the eikonal equation (3.1).

4. $\operatorname{Graph}(\Omega)$ is open in $[0, T] \times \mathbb{R}^{N}$ and $\bar{u}:(x, t) \mapsto \mathbf{1}_{\Omega(t)}(x)$ is a supersolution of the eikonal equation (3.1).

5. $\mathcal{L}^{N+1}(\operatorname{Graph}(K) \backslash \operatorname{Graph}(\Omega))=0$.

Then for a.a. $t \in[0, T], K(t)$ has locally finite perimeter in $\{c(\cdot, t) \neq 0\}$, and if $\operatorname{sgn}(r)$ denotes the sign of $r \in \mathbb{R}(\operatorname{sgn}(r)=r /|r|$ if $r \neq 0, \operatorname{sgn}(0)=0)$, we have the following estimate: 


$$
\begin{aligned}
& \int_{0}^{T} \int_{\partial^{*} K(t)}|c(x, t)| \mathrm{d} \mathcal{H}^{N-1}(x) \mathrm{d} t \\
& \quad \leqslant\left[\int_{K(t)} \operatorname{sgn}(c(x, t)) \mathrm{d} x\right]_{0}^{T}+2 \int_{0}^{T} \int_{K(t) \cap\{c(\cdot, t)=0\}} \frac{\left|c_{t}(x, t)\right|}{|D c(x, t)|} \mathbf{1}_{\left\{c_{t}<0\right\}}(x, t) \mathrm{d} \mathcal{H}^{N-1}(x) \mathrm{d} t .
\end{aligned}
$$

REMARK 7.2 The existence of $\Omega$ and $K$ satisfying assumptions 1,3 and 4 is ensured by Theorem 2.2 and the paragraph that follows it. Assumption 2 avoids pathological behavior of the front at times 0 and $T$, and assumption 5 is related to the so-called non-empty interior difficulty (see [9]).

Proof. Since $\mathcal{L}^{N+1}(\operatorname{Graph}(K) \backslash \operatorname{Graph}(\Omega))=0$, we have $\mathcal{L}^{N}(K(t) \backslash \Omega(t))=0$ for almost all $t \in[0, T]$. Therefore $K(t)$ has locally finite perimeter in $\{c(\cdot, t) \neq 0\}$ for almost all $t$ such that $K(t)$ has locally finite perimeter in $\{c(\cdot, t)<0\}$ and $\Omega(t)$ has locally finite perimeter in $\{c(\cdot, t)>0\}$, and then $\partial^{*} \Omega(t)=\partial^{*} K(t)$. Finally, using the fact that $|c|=c_{-}+c_{+}$, we deduce the global estimate by summing the estimates of Theorems 5.1 and 6.4 .

\section{Acknowledgements}

My grateful thanks go to Pierre Cardaliaguet for introducing me to the subject of viscosity solutions, and for fruitful discussions during the preparation of this article. I also thank Régis Monneau for pointing out that one of the original assumptions in Theorem 4.1 was not necessary.

\section{REFERENCES}

1. Alvarez, O., Cardaliaguet, P., \& Monneau, R. Existence and uniqueness for dislocation dynamics with nonnegative velocity. Interfaces Free Bound. 7 (2005), 415-434. Zbl 1099.35148 MR 2191694

2. Ambrosio, L., \& Dancer, N. Calculus of Variations and Partial Differential Equations. Topics on geometrical evolution problems and degree theory, Papers from the Summer School held in Pisa, September 1996, Springer, Berlin (2000). MR 1757706

3. Ambrosio, L., Fusco, N., \& Pallara, D. Functions of Bounded Variations and Free Discontinuity Problems. Oxford Math. Monogr., Oxford Univ. Press, New York (2000). MR 1857292

4. Aubin, J.-P., \& Frankowska, H. Set-Valued Analysis. Systems Control Found. Appl. 2, Birkhäuser Boston, Boston, MA (1990). Zbl 0713.49021 MR 1048347

5. BARdi M., \& CApuzzo-DolcetTA, I. Optimal Control and Viscosity Solutions of HamiltonJacobi-Bellman Equations. Systems Control Found. Appl., Birkhäuser Boston, Boston, MA (1997). Zbl 0890.49011 MR 1484411

6. Bardi M., Crandall, M. G., Evans, L. C., Soner, H. M., \& Souganidis, P. E. Viscosity Solutions and Applications. Lecture Notes in Math. 1660, Springer, Berlin (1995). Zbl 0884.49020 MR 1462700

7. BARles, G. Solutions de viscosité des équations de Hamilton-Jacobi. Springer, Paris (1994). Zbl 0819.35002 MR 1613876

8. BARLES, G., \& LEY, O. Nonlocal first-order Hamilton-Jacobi equations modelling dislocations dynamics. Comm. Partial Differential Equations 31 (2006), 1191-1208. Zbl pre05062574 MR 2254611

9. Barles, G., Soner, H. M., \& Souganidis, P. E. Front propagation and phase field theory. SIAM J. Control Optim. 31 (1993), 439-469. Zbl 0785.35049 MR 1205984

10. Bellettini G., \& Novaga, M. Minimal barriers for geometric evolutions. J. Differential Equations 139 (1997), 76-103. Zbl 0882.35028 MR 1467354 
11. Cannarsa, P., \& Cardaliaguet, P. Perimeter estimates for reachable sets of control systems. $J$. Convex Anal. 13 (2006), 253-267. Zbl pre05046589 MR 2252231

12. Cannarsa, P., \& Sinestrari, C. Semiconcave Functions, Hamilton-Jacobi Equations, and Optimal Control. Progr. Nonlinear Differential Equations Appl. 58, Birkhäuser Boston, Boston, MA (1990). Zbl pre02129788 MR 2041617

13. CRANDAll, M. G., IshiI, H., \& LiONs, P.-L. User's guide to viscosity solutions of second order partial differential equations. Bull. Amer. Math. Soc. 27 (1992), 1-67. Zbl 0755.35015 MR 1118699

14. Crandall, M. G., \& Lions, P.-L. Viscosity solutions of Hamilton-Jacobi equations. Trans. Amer. Math. Soc. 277 (1983), 1-42. Zbl 0599.35024 MR 0690039

15. Evans, L. C., \& GariePy, R. F. Measure Theory and Fine Properties of Functions. Stud. Adv. Math., CRC Press, Boca Raton, FL (1992). Zbl 0804.28001 MR 1158660

16. Evans, L. C., \& Spruck, J. Motion of level sets by mean curvature, IV. J. Geom. Anal. 5 (1995), 77-114. Zbl 0829.53040 MR 1315658

17. Giga, Y. Surface Evolution Equations. A Level Set Approach. Monogr. Math. 99, Birkhäuser (2006). Zbl pre05014137 MR 2238463

18. Jensen, R., Lions, P.-L., \& Souganidis, P. E. A uniqueness result for viscosity solutions of secondorder fully nonlinear pde's. Proc. Amer. Math. Soc. 102 (1988), 975-978. Zbl 0662.35048 MR 0934877

19. Lions, P.-L. Generalized Solutions of Hamilton-Jacobi Equations. Res. Notes in Math. 69, Pitman, Boston, MA (1982). Zbl 0497.35001 MR 0667669

20. Rockafellar, R. T., \& Wets, R. J.-B. Variational Analysis. Grundlehren Math. Wiss. 317, Springer, Berlin (1998). Zbl 0888.49001 MR 1491362

21. Soner, H.M. Motion of a set by the curvature of its boundary. J. Differential Equations 101 (1993), 313-372. Zbl 0769.35070 MR 1204331 